Arts

et Savoirs
Arts et Savoirs

9 | 2018

Ernst Haeckel entre science et esthétique

\title{
Jules Soury (1842-1915), traducteur de Ernst Haeckel
}

\section{Laura Bossi}

\section{(2) OpenEdition \\ 1 Journals}

Édition électronique

URL : http://journals.openedition.org/aes/1168

DOI : $10.4000 /$ aes. 1168

ISSN : 2258-093X

\section{Éditeur}

Laboratoire LISAA

Référence électronique

Laura Bossi, « Jules Soury (1842-1915), traducteur de Ernst Haeckel », Arts et Savoirs [En ligne],

9 | 2018, mis en ligne le 14 mai 2018, consulté le 01 mai 2019. URL : http://journals.openedition.org/ aes/1168; DOI : 10.4000/aes.1168

Ce document a été généré automatiquement le 1 mai 2019.

Centre de recherche LISAA (Littératures SAvoirs et Arts) 


\title{
Jules Soury (1842-1915), traducteur de Ernst Haeckel
}

\author{
Laura Bossi
}

Nous remercions très chaleureusement Olaf Breidbach ( $\dagger$ ) et Thomas Bach, du Ernst-Haeckel-Haus de Iéna, pour la permission de publier les lettres de Soury. Nous remercions aussi Margot Georges, de la bibliothèque de l'EPHE, IV ection, pour son aide dans la consultation des archives Soury. 
1 Le zoologue allemand Ernst Haeckel (1834-1919) est connu surtout comme l'apôtre du darwinisme en Allemagne ${ }^{1}$; mais son rôle dans l'histoire des sciences et dans l'histoire des idées reste sous-estimé. En France en particulier, il reçut un accueil qu'on a pu qualifier de «triomphal $»^{2}$.

2 Entre 1874 et 1907, onze ouvrages de Haeckel sont publiés en traduction française $^{3}$, dont neuf chez l'éditeur d'origine allemande Carl Reinwald et deux à la maison d'édition scientifique GermerBaillière. Il s'agit des principales œuvres

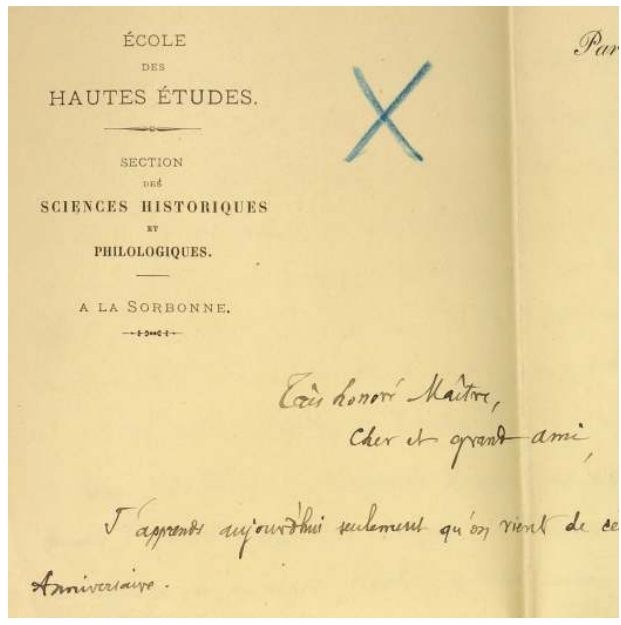
destinées au "grand public » ( gemeinverständliche Werke); ses œuvres plus théoriques ou techniques, adressées aux scientifiques, comme le traité sur les radiolaires ${ }^{4}$, la Generelle Morphologie (Morphologie générale), ou encore Die Kalkschwämme (Les Éponges calcaires), n'ont jamais été traduites.

Les traducteurs français de Haeckel seront tous liés au milieu rationaliste, matérialiste, positiviste, scientiste, républicain et anticlérical associé à ces maisons d'édition, ou à la Société d'anthropologie de Paris, à l'époque un foyer de la libre pensée ${ }^{5}$. On notera aussi qu'aucun de ses traducteurs n'est zoologue ou biologiste, à la différence des traducteurs d'autres pays comme l'Angleterre ou l'Italie.

Parmi les traducteurs français d'Ernst Haeckel, l'« étonnant, admirable et funeste ${ }^{6}$ » Jules Soury (28 mai 1842-10 août 1915) occupe une place singulière. L'œuvre de Haeckel fut capitale pour Soury, qui l'assimila et l'incorpora dans sa vision du monde et dans ses propres œuvres d'histoire de la " psychologie physiologique ».

Fils d'un petit artisan opticien parisien, largement autodidacte, puis chartiste érudit, Soury débuta sa carrière intellectuelle comme disciple et proche d'Ernest Renan, qui l'initia à la langue hébraïque, à l'exégèse biblique, à la critique des religions, et l'introduisit à la Revue des Deux Mondes et au Temps ${ }^{7}$. En 1865, il fut embauché comme bibliothécaire à la Bibliothèque nationale (alors Bibliothèque impériale). En parallèle, dès 1865, il suivit, à la Salpêtrière, l'enseignement de deux éminents neuropsychiatres : JulesBernard Luys (1828-1897) ${ }^{8}$, et Auguste-Félix Voisin (1829-1898) ${ }^{9}$; il deviendra ainsi l'un des premiers et des rares historiens de la psychologie avec une connaissance approfondie et de première main de l'anatomie et de la physiologie du cerveau.

En 1878 Soury publia des Essais de critique religieuse, dans lesquels il affichait un athéisme militant ${ }^{10}$ et son Jésus et les Évangiles ${ }^{11}$ (1878), écrit clairement dans la filiation des Vies de Jésus de David Friedrich Strauß (1808-1874) ${ }^{12}$ et de Renan, était un véritable pamphlet antichrétien. Si Strauß avait analysé les Évangiles comme un ensemble de mythes, et Renan présenté Jésus comme un « homme admirable » et traité sa vie comme un roman, Jules Soury décrivait Jésus comme un cas pathologique, un « dégénéré épileptique » ${ }^{13}$.

7 C'est cette même année qu'il contacta Haeckel à l'occasion du séjour de ce dernier à Paris. Il allait traduire trois ouvrages de Haeckel entre 1879 et $1880^{14}$ et introduire celui-ci chez l'éditeur Germer-Baillière.

8 Soury collabora à de nombreux journaux et revues, dont la Revue scientifique dirigée par le physiologiste (élève de Claude Bernard) et homme politique Paul Bert (1833-1886). C'est 
celui-ci qui obtint pour lui un poste à l'École Pratique des Hautes Études ${ }^{15}$, le 30 novembre 1881. Il y enseigna, de 1882 et jusqu'à sa mort en 1915, l'Histoire des doctrines de psychologie physiologique contemporaines. C'est à partir de ses cours qu'il rédigera son opus magnum, Le Système nerveux central (1899) ${ }^{16}$, somme d'une érudition telle qui fait que Jules Soury peut être considéré comme l'un des tout premiers historiens de la neurologie ${ }^{17}$. Le personnage du philosophe Adrien Sixte dans Le Disciple ${ }^{18}$ de Paul Bourget est sans doute inspiré de Soury. Le "maitre » du déterminisme biologique, «emprisonné dans l'atmosphère des spéculations abstraites", auquel le jeune protagoniste Robert Greslou adresse sa confession, enseigne la psychologie comparée à l'École des Hautes Études, a un père horloger, a étudié en autodidacte la physiologie du cerveau, a publié un premier ouvrage scandaleusement athée, mène une vie monacale toute dédiée à la pensée...

Célèbre en son temps, Soury en vient à représenter « le chef incontesté de la philosophie naturelle »; les contemporains le comparent aux géants de la génération précédente, Renan et Taine. Il est considéré comme le maître à penser de Maurice Barrès ${ }^{19}$.

Il se définissait comme un "athée clérical ${ }^{20}$, souscrivant à la tradition catholique au nom d'un culte des ancêtres fondé sur un déterminisme biologique absolu, et sur une obsession de l'hérédité qui sans doute doit beaucoup à ses lectures haeckeliennes. Au moment de l'affaire Dreyfus, ses écrits d'un antisémitisme racialiste virulent l'éloignèrent de ses collègues de la Sorbonne et de la plupart de ses amis. Après sa mort en 1915, il sombra dans l'oubli.

Les archives du Ernst-Haeckel-Haus à Iéna conservent trente-cinq lettres inédites adressées par Soury à Haeckel entre 1878 et 1914, qui documentent la relation des deux hommes. Elles permettent également de suivre quelques étapes clés de la vie de Soury, et confirment par ailleurs l'interprétation rigidement mécaniste, matérialiste et athée des théories haeckeliennes par Soury, déjà évidente dans les longues préfaces qu'il rédige pour ses traductions.

\section{La rencontre d'Ernst Haeckel et Jules Soury}

12 Lorsque Soury entreprend ses traductions de Haeckel, celui-ci est déjà connu et apprécié en France. Le philosophe Léon Dumont (1837-1877) avait été le premier à le faire découvrir au public français par son ouvrage paru en 1873 à Paris chez Germer-Baillière, Haeckel et la théorie de l'évolution en Allemagne, qui s'appuie sur la quatrième édition allemande de la Natürliche Schöpfungsgeschichte. Celle-ci sera traduite l'année suivante par l'anthropologue Charles-Jean-Marie Letourneau (Histoire de la création), qui traduira aussi l'Anthropogenie $e^{21}$.

13 L'ouvrage de Dumont et les deux traductions de Letourneau exposent les principales doctrines de Haeckel, et ce n'est pas le lieu de les résumer en détail. Rappelons seulement qu'il attribue trois fondateurs à la théorie de l'évolution : Goethe aurait été le premier à proposer une interprétation mécanique de la communauté originelle comme des métamorphoses des formes animales et végétales, et aurait aussi exprimé l'idée d'une parenté généalogique entre les plantes et les animaux ${ }^{22}$; Lamarck serait le véritable père de la théorie de la descendance commune des espèces; Darwin aurait assigné au transformisme une cause scientifique, une "explication mécanique», avec la sélection naturelle. Haeckel, quant à lui, se pose comme le quatrième fondateur. Il se propose en effet non seulement de «compléter» le darwinisme par son application à l'homme ${ }^{23}$, 
absente dans l'Origine des espèces, et par l'intégration du monde vivant dans l'évolution cosmique, avec le postulat d'une "autogonie ", la formation d'organismes très simples par génération spontanée; il tente aussi de donner une explication «mécanique » des phénomènes qui interviennent dans l'évolution : l'hérédité, la reproduction, la variation, et l'adaptation ${ }^{24}$. L'hérédité conservatrice relèverait de la «loi biogénétique fondamentale » selon laquelle l'ontogénèse récapitule la phylogénèse ${ }^{25}$; l'hérédité progressive serait la faculté de léguer des propriétés acquises pendant sa vie, par l'influence du milieu ambiant. Surtout il entreprend le premier, de manière audacieuse, de retracer les métamorphoses de la phylogénie et de reconstituer les relations généalogiques de l'ensemble des êtres organiques, de la monère jusqu'à l'homme ${ }^{26}$. Enfin, il propose un système de philosophie naturelle, le monisme, selon lequel le monde est constitué par une substance unique, qui est en même temps matière et esprit, et qui est régie par les lois de la mécanique ${ }^{27}$.

Soury est à cette époque très actif comme journaliste scientifique au Temps et à la République française; formé à l'étude du système nerveux à l'École de la Salpêtrière, il est manifestement au courant de la littérature scientifique dans les domaines de l'anatomie comparée et de la physiologie. En 1873 il avait publié une longue critique de l'œuvre d'Hippolyte Taine, De l'intelligence (1870), montrant son extrême réductionnisme: il affirme que les causes de l'intelligence doivent être recherchées dans « la substance grise des circonvolutions cérébrales", et reproche à Taine de ne pas avoir consulté les Recherches sur le système nerveux spinal de Jules Luys ${ }^{28}$. Par ailleurs, il a déjà fait ses preuves comme traducteur de l'allemand, avec des traductions de l'orientaliste Theodor Nöldeke, de l'ophtalmologiste Hugo Magnus, et du naturaliste Eduard Oscar Schmidt, darwiniste et ami de Haeckel ${ }^{29}$. Il est donc tout à fait compréhensible qu'il s'intéresse à l'œuvre de Haeckel, et qu'il l'approche pour lui demander de le traduire.

L'année 1878, avec un corpus de 14 lettres envoyées par Soury à Haeckel, est celle de la « lune de miel ». Dans un premier groupe de 8 lettres rédigées entre le 25 février et le 22 août 1878 , Soury « fait sa cour ». Se présentant comme « journaliste et bibliothécaire », il lui propose de rédiger des comptes-rendus de ses travaux dans la presse française et de traduire ses ouvrages. Il signe modestement «votre toujours dévoué et respectueux disciple et admirateur ». Lorsque Haeckel se rend à Paris en août 1878 pour le Congrès de l'Association française pour l'avancement de la science ${ }^{30}$, Soury fait partie du petit comité de libres penseurs qui propose d'organiser un banquet en son honneur :

Mes amis et moi, MM. les docteurs E. Meyer, G. le Bon, Dally, $\mathrm{M}^{\mathrm{e}}$ Clémence Royer, Onimus, A. Gout, M. Reinwald aussi, sans doute, ainsi que M. van Grevens [...], nous désirerions nous rencontrer avec vous chez Breban ${ }^{31}$ ou dans les salons du Grand Hôtel, le soir qui vous conviendrait le mieux, en un mot, nous voudrions vous recevoir comme on a reçu naguère le philosophe Herbert Spencer. ${ }^{32}$

Lors de ce banquet, qui a lieu au Grand Hôtel, Soury prononce le discours de bienvenue, ce qui lui vaut d'être qualifié d'« ennemi personnel de Jésus Christ» dans un article du Figaro du 30 août ; les discours de Soury et de Haeckel sont publiés dans Le Temps ${ }^{33}$. À la suite de cette rencontre, Haeckel est convaincu, et confie à Soury la traduction française de quatre essais, qui donneront lieu à trois livres. Dans un ensemble de 5 lettres (datées du 20 septembre au 11 novembre 1878), Soury confirme la réception des quatre essais de Haeckel qu'il va traduire, et propose de réunir Zellenseelen und Seelenzellen et Die Perigenesis der Plastidule en un seul volume. 


\section{Les trois ouvrages traduits par Jules Soury}

17 Le premier ouvrage de Haeckel traduit par Soury, Les Preuves du transformisme $e^{34}$ (1879), est un texte militant : il s'agit de la réponse de Haeckel à Rudolf Virchow, à la suite de leur querelle éclatée lors du cinquantième congrès des naturalistes et médecins allemands $s^{35}$. Virchow s'était vivement opposé à Haeckel sur le statut du transformisme (qu'il tient pour une simple « hypothèse non prouvée ») et sur l'opportunité de l'enseigner dans les écoles $^{36}$, mais aussi sur les théories plus proprement haeckeliennes des «âmes cellulaires » (présence chez les organismes unicellulaires d'une vie " psychique ", à savoir de sensation, perception, volonté, mouvement, et conception "moniste» de l'âme humaine comme la somme d'un nombre d'activités ou de fonctions cellulaires spéciales) et de la mémoire des plastidules ${ }^{37}$.

Haeckel s'interroge : « Comment est-il possible qu'un homme qui a été longtemps à la tête du parti progressiste, dans la science comme dans la politique, et qui même n'a pas cessé de l'être dans celle-ci, soit devenu au contraire, dans celle-là, l'instrument de la plus dangereuse réaction? » Son ancien maître Virchow, éminent scientifique fondateur de la pathologie cellulaire, est ainsi présenté comme un «libre penseur converti à l'obscurantisme ", comme le partisan d'une création surnaturelle des espèces, ou comme un ignorant et un incompétent, devenu étranger aux progrès de la science nouvelle à cause de ses engagements politiques ${ }^{38}$

Haeckel définit d'abord brièvement les trois grandes théories que, à son avis, Virchow confond: le monisme, ou théorie générale de l'évolution, ou conception mécanique du monde ; la théorie de la descendance, ou transformisme, ou lamarckisme ; et la théorie de la sélection, ou darwinisme, que Haeckel présente comme la plus importante parmi les théories qui cherchent à expliquer par des causes mécaniques la transformation des espèces. Il entreprend ensuite d'énumérer les preuves du transformisme et de la descendance simienne de l'homme, dans le domaine de la morphologie et de la physiologie comparée, surtout des animaux inférieurs, de la craniologie, etc., donnant de la sorte un abrégé de ses principales doctrines. Mais c'est aussi toute son approche de l'enseignement que Haeckel défend. Contre Virchow pour qui «nous devons soigneusement distinguer entre l'enseignement et la recherche. Ce que nous cherchons, ce sont des problèmes. Mais le problème ne doit pas faire l'objet immédiat de l'enseignement $»^{39}$, Haeckel rappelle que l'enseignement de Virchow lui-même avait été remarquable et passionnant justement parce qu'il livrait toujours des hypothèses, des problèmes à résoudre, et non des faits, sûrs, indubitables; et il conclut avec un vibrant plaidoyer pour la liberté de la science et de son enseignement ${ }^{40}$.

Ce court pamphlet de Haeckel (142 pages) est précédé d'une préface du traducteur de 32 pages. Soury ne se limite pas à situer l'essai de Haeckel dans le contexte de la querelle, ou à en discuter les arguments; il prend résolument parti pour le point de vue moniste, «l'explication mécanique qui découvre les causes dernières des phénomènes dans les mouvements des particules ultimes de la matière ", l'origine simienne de l'homme, la génération spontanée des monères primitives dans la mer, "comme les cristaux salins naissent dans les eaux-mères ». Il présente aussi, d'une manière très claire, d'autres doctrines que Haeckel traite surtout dans d'autres ouvrages, comme la théorie de la récapitulation « pour laquelle l'évolution embryologique individuelle que Haeckel appelle ontogénie, doit être un résumé rapide, une brève récapitulation de l'évolution 
paléontologique, de la longue existence des espèces antérieures, de la phylogénie $»^{41}$; ou la théorie de la gastrée, exposée pour la première fois dans la Monographie des éponges calcaires (1872), selon laquelle le premier ancêtre de tous les animaux devait ressembler à la gastrula, constituée de deux feuillets germinatifs. Mais dans ce long chemin qui mène de la monère à l'homme, c'est surtout le développement du cerveau qui intéresse Soury :

De même que le développement intellectuel de chaque enfant n'est qu'une brève répétition de l'évolution mentale qui, à travers des milliers d'années, a fait sortir l'esprit humain de l'intelligence rudimentaire des autres vertébrés, l'embryologie du système nerveux est un rapide abrégé de l'évolution historique par laquelle a passé l'organe de l'âme, de l'humble ganglion sus-œsophagien des vers au cerveau si complexe des singes anthropoïdes et de l'homme ${ }^{42}$. [...] On entrevoit maintenant ce que sera la psychologie de l'avenir, quand, au lieu d'étudier l'homme blanc adulte, l'Européen civilisé de Paris ou de Londres, elle fondera ses spéculations sur les principes généraux de l'anatomie comparée, de la physiologie et de l'embryologie du système nerveux..$^{43}$

Et il conclut :

Des livres comme la Morphologie générale, l'Histoire naturelle de la création, l' Anthropogénie et Les Preuves du transformisme - sorte de synthèse ou de résumé philosophique de la doctrine, - ne paraissent pas en vain dans le monde. Ils annoncent une rénovation prochaine de la pensée, un renouvellement lent, mais fatal, de la conscience, une évolution de l'esprit humain. ${ }^{44}$

Le 21 juin 1879, Soury envoie à Haeckel sa recension de deux de ses essais, parue dans $L a$ Revue scientifique; il annonce qu'il travaille sur la préface du Règne des protistes, et qu'il lui a trouvé un précurseur français en la personne de Bory de Saint-Vincent. Avec cette deuxième traduction ${ }^{45}$, Soury s'attaque à un ouvrage d'un abord plus difficile : il s'agit d'un aperçu de la morphologie et d'une tentative de classification des êtres unicellulaires ou protistes ${ }^{46}$, que Haeckel propose de placer dans un règne indépendant du règne animal et du règne végétal.

Le 24 juin, Soury s'inquiète de la menace de l'éditeur Reinwald de supprimer sa préface : "Dites à l'éditeur allemand [...] d'attendre la préface au Règne des protistes qui est nécessaire car le public français, même cultivé, est tout à fait étranger à vos études de biologie.» Le 27 juillet la préface est finalement terminée, et Soury demande une intervention de Haeckel car «l'éditeur allemand» veut la réduire à un maximum de quatre pages.

L'intervention a apparemment été efficace, car la préface est maintenue (60 pages, pour un essai de 121 pages...). Soury rend d'abord hommage à la « haute valeur philosophique » de ces organismes :

Si un rayon de lumière est tombé naguère sur l'obscur et vaste empire des Protozoaires, sur ce " chaos systématique ", c'est surtout à M. Haeckel qu'on le doit, grâce à sa découverte des Monères. Ces organismes, les plus simples peut-être qu'on peut imaginer, puisqu'ils n'ont pas d'organes, pourraient être, avec autant de droit, c'est-à-dire avec aussi peu de raison, considérés comme des rudiments d'organisation animale ou végétale. La haute valeur philosophique de cet état d'indifférence morphologique, chez des êtres qui vivent encore sur cette planète, ne pouvait échapper au naturaliste qui, après Lamarck et Darwin, aura le plus contribué à fonder dans le domaine des sciences biologiques la théorie de l'évolution. D'autres considérations, qu'on lira en ce livre, l'inclinèrent à faire entrer ces êtres, avec les Rhizopodes, les Amibes, les Diatomées, les Flagellés etc., dans un règne destiné à réunir, comme une zone frontière, les deux grands règnes des végétaux et des animaux, en même temps qu'à leur servir de fondement. 
Mais, loin d'avoir voulu élever je ne sais quelles murailles de la Chine entre les plantes, les animaux et les protistes, M. Haeckel a maintes fois témoigné qu'il tenait plutôt pour très vraisemblable que les animaux, aussi bien que les végétaux, descendent de certains Protistes, et, en particulier, des plus simples de ceux-ci, les Monères, tandis que d'autres groupes de Protistes (Diatomées, Myxomycètes, Rhizopodes) se sont développés isolément. Cette division systématique des trois règnes organiques n'a jamais eu à ses yeux qu'un but pratique : faciliter la diagnose différentielle des corps organisés. ${ }^{47}$

En bon patriote, Soury s'emploie ensuite à présenter les savants français ayant traité du même sujet, qu'ils soient "précurseurs " ou contemporains de Haeckel. Bory de Saint Vincent (1778-1846), «l'un des disciples du grand Lamarck, le père du Règne psychodiaire », "précurseur» de Haeckel, a droit à un chapitre entier, bien que son «règne psychodiaire " ne conserve qu'un intérêt historique ${ }^{48}$.

Dans les deux chapitres suivants, traitant de l'«Origine et nature de la vie», et de l'« Unité de la vie dans les trois règnes organiques », c'est Claude Bernard (1813-1878), qui venait de mourir le 10 février 1878, qui est cité à plusieurs reprises, et que Soury tente de confronter et de rapprocher de Haeckel. Comme Haeckel, Claude Bernard aurait une vision unitaire de la nature et des sciences :

Comment pourrions-nous comprendre, a dit éloquemment Claude Bernard, un antagonisme, une opposition entre les propriétés des corps vivants et celles des corps bruts, puisque les éléments constituants de ces deux ordres de corps sont les mêmes? Tous les corps vivants sont exclusivement formés d'éléments minéraux, empruntés au milieu cosmique. Descartes, Leibnitz, Lavoisier, nous ont appris que la matière et ses lois ne diffèrent pas dans les corps vivants et les corps bruts; ils nous ont montré qu'il n'y a au monde qu'une seule mécanique, une seule physique, une seule chimie, commune à tous les êtres de la nature..$^{49}$

Soury énumère ensuite nombre d'arguments de Claude Bernard tendant à montrer comme les phénomènes de la vie, respiration, reproduction, mouvement, sensibilité, peuvent être ramenés à des phénomènes physico-chimiques. «Rien là qui soit spécial au monde vivant [...] : la nature physique obéit à la même règle. $\|^{50}$ Puis, Soury rappelle que Claude Bernard, dans ses cours au Muséum, enseignait qu' i il n'y a qu'une seule manière de vivre, qu'une seule physiologie pour tous les êtres vivants " ${ }^{51}$. Cette " unité vitale dans les deux règnes ", Claude Bernard la voyait jusque dans la nutrition: « Si l'on définit la nutrition un perpétuel échange de liquides et de gaz entre l'élément anatomique et le milieu ambiant, on voit de reste qu'il n'est pas de phénomène plus essentiel à tous les êtres vivants, à quelque règne qu'ils appartiennent. $\|^{52}$ La respiration est identique dans les trois règnes. "Quant à la sensibilité, [...] c'est une propriété générale de la matière. ॥ ${ }^{53}$ Le protoplasma est susceptible d'être sollicité par le milieu interne ou externe, et de réagir à cette excitation. "L'eau, la chaleur, l'oxygène, les substances dissoutes dans le milieu ambiant, c'est-à-dire, en somme, Claude Bernard en a fait la remarque, ce que les anciens appelaient les quatre éléments, voilà les sources des excitations extérieures. $»^{54}$ Le mouvement n'est pas non plus spécifique aux animaux, car des spores et des plantes se meuvent au moyen de filaments, de cils vibratiles. Enfin, Claude Bernard aurait approuvé l'idée d'un protoplasme amorphe dans lequel résiderait la vie, la vie non encore définie, et l'adoption d'un troisième règne organique, de « ces êtres dont on ne peut dire s'ils sont animaux ou végétaux $»^{55}$. Il n'y aurait pas jusqu'à la théorie plastidulaire de la mémoire que Claude Bernard n'ait paru considérer d'un œil favorable, lui qui avait écrit que «le germe semble garder la mémoire de l'organisme dont il procède. $»^{56}$ 
Le troisième ouvrage traduit par Soury, Essais de psychologie cellulaire ${ }^{57}$ (1880), réunit deux essais de Haeckel : La Périgenèse des plastidules (Die Perigenesis der Plastidule), qui contient l'une de toutes premières théories de la mémoire héréditaire, une mémoire inconsciente des molécules du plasson, les plastidules, qui transmetteraient de génération en génération leurs propriétés caractéristiques dans un mouvement rythmique continu; et Psychologie cellulaire (Zellseelen und Seelenzellen), décrivant l'appareil psychique ou « organe de l'âme" comme un ensemble composé de millions d'âmes indépendantes, chaque cellule cérébrale étant un organisme élémentaire, doué d'une forme, d'une vie, et aussi d'une "âme", une âme cellulaire propre. Les deux essais, de 158 pages en tout, sont précédés d'une préface du traducteur de 25 pages. En voici quelques extraits, qui montrent que Soury interprète l'hylozoïsme de Haeckel comme un monisme "strictement mécanique ", et n'hésite pas à critiquer l'usage du terme «âme ", qui lui paraît dangereusement spiritualiste, et dont Haeckel n'aurait pas voulu se débarrasser par crainte de « se couper de toutes les sources poétiques du passé » :

Un nombre considérable de propriétés regardées comme particulières à la matière vivante ou animée, la sensibilité elle-même, se sont trouvées appartenir aussi bien, quoique à de degrés très différents, à la matière brute. De là à croire aux « forces psychiques » et aux « âmes » des atomes et des plastidules, il n'y avait qu'un fossé à franchir, encore assez large pourtant ; Haeckel l'a franchi ; je ne le suivrai pas, de peur d'une chute.

Je ne saurais admettre, en effet, que les atomes possèdent des «forces »; qu'ils éprouvent du plaisir et du déplaisir, des amours et des haines lorsqu'ils se joignent ou se repoussent; qu'ils agissent à distance, sentent et veulent; bref, qu'ils aient une " âme ». Ce dernier vocable revient trop souvent dans ce livre; il étonne; la psychologie de l'avenir sera sûrement une "psychologie sans âme». Et, comme il arrive, nul n'aura plus contribué à la fonder que l'auteur de la Psychologie cellulaire. [...] Loin de ressembler à un vague panthéisme, son monisme est strictement mécanique. C'est même un peu, j'imagine, pour échapper à la difficulté de faire commencer «l'âme » à un moment de la durée, qu'il l'a faite éternelle, comme l'atome..$^{58}$

Aussi, sans méconnaître l'intérêt de la théorie de la périgenèse des plastidules, Soury ne se prive pas de la critiquer :

Seules, les molécules du plasson, les plastidules ont de la mémoire. Pourquoi? Haeckel ne le dit pas. Mais ne pourrait-il pas répondre que cette propriété apparait ici pour la première fois grâce à une combinaison nouvelle de la matière, à la combinaison dont sont formées les molécules du plasson?

Dans une longue lettre sans date (mais sans doute rédigée entre le 26 Juillet et le 16 décembre 1879) Soury annonce à Haeckel la parution et l'envoi d'un exemplaire de la Psychologie cellulaire, qu'il espère appelée à avoir « un grand retentissement dans toute la France ", " grâce à la grande publicité dont dispose la librairie Germer-Baillière et Cie " ${ }^{59}$.

S'il loue les théories de Haeckel sur les propriétés psychiques des cellules et même du monde inorganique, et sur la périgenèse des plastidules, il rappelle sa réticence vis-à-vis du terme « âme », « cette abstraction ne faisant qu'embrouiller et compliquer le problème biologique dont il s'agit». Il tient ces théories pour «le fondement véritable de toute psychologie de l'avenir, laquelle devra être une psychologie sans âme ».

31 De fait, après ces trois traductions coup sur coup, la carrière de Soury connaît un tournant qui l'amènera à enseigner la "psychologie physiologique " et à devenir le premier historien de la neurologie. 


\section{Les vicissitudes d'une relation intellectuelle} obtenir une chaire d'histoire comparée des religions au Collège de France, à cause de l'opposition de Jules Ferry (1832-1893), alors ministre de l'Instruction publique, et de M. de Laboulaye, administrateur du Collège de France. Cette étrange aventure est décrite par Renan dans une lettre :

Nous possédons dans notre littérature l'hircocerf le plus étrange que jamais la nature ait produit ; c'est Jules Soury. Dons de nature surprenants; le grand style inné; prodigieuse facilité d'assimilation des idées d'autrui ; esprit éminemment philosophique ; puis tout cela gâté par une déplorable étourderie, un amour-propre insupportable, un manque de sérieux désolant. Enfin corruptio optima, c'est le seul être créé qui ait réussi quelquefois à me faire sortir de mes gonds.

Soury écrit dans $L a$ République française sans appartenir au parti démocratique. Gambetta remarqua ses articles, attacha plus d'importance qu'il ne fallait à leur valeur de fond, mais il ne se trompa pas sur le talent. Il fit venir Soury dont la situation à la Bibliothèque Nationale est fort inférieure, et lui dit : « II vous faut une situation, une chaire, que pourrait-on faire? » Soury eut l'idée du monde la plus saugrenue, du moins quant à la forme. Il proposa une chaire d'éthologie ou mœurs comparées des peuples. Gambetta répondit que jamais commission du budget ne comprendrait cela. Soury dit alors : «Et une chaire de religions comparées? » Le mot fut saisi avec empressement. Une telle idée flattait l'anticléricalisme de Gambetta et d'ailleurs était mise en avant par Paul Bert (esprit ardent et puissant mais très hasardé dans la pratique) presque dans les mêmes termes, comme un moyen de remplacer toutes les facultés de théologie. ${ }^{61}$

Après cet échec, qu'il vit comme une grande injustice et un affront personnel, Jules Soury s'applique à étoffer ses qualifications académiques, et prépare sa double thèse de doctorat ès lettres. Dans cette perspective, il écrit à Haeckel le 6 février 1880, pour lui demander des références bibliographiques sur l'hylozoïsme: "Je connais Glisson, Leibnitz (les monades ne sont que des atomes doués de perception et d'appétition), Maupertuis (la thèse du Dr. Baumann), Robinet et... Haeckel. Mais bien d'autres ont dû traiter cette matière... ». Dans une lettre du 17 septembre 1881, il se plaindra auprès de Haeckel de ne pas avoir eu «sa » chaire au Collège de France : «Et à moi non plus, dans mon humble sphère, on ne me permet pas d'être "Atheist, Monist et Darwinist"; on ne m'a pas encore rendu la chaire qui m'a été promise et qu'on me doit. »

Soury soutient ses deux thèses en Sorbonne en 1881. La thèse française, Théories naturalistes du monde et de la vie dans l'Antiquité, est dédiée à Ernest Renan, la thèse latine, De hylozoismo apud recentiores, à l'helléniste Émile Egger $^{62}$; elles seront publiées dans une forme revue et augmentée la même année ${ }^{63}$.

Peu après, Paul Bert devient à son tour ministre de l'Instruction publique et des Cultes : s'il ne peut donner à son ami la chaire promise, il lui fait cependant obtenir une charge de cours à la IV e section (Sciences historiques et philologiques) de l'École Pratique des Hautes Études, le 30 novembre $1881^{64}$. Jules Soury y enseignera, de 1882 et jusqu'à sa mort en 1915, l'Histoire des doctrines de psychologie physiologique contemporaines.

Son enseignement, nouveau et original, remportera un grand succès. En 1891 Anatole France fera de lui un portrait enthousiaste dans sa rubrique du Temps, où il est présenté un scalpel à la main, un cerveau sur la table, enseignant la théorie des localisations cérébrales : 
C'est là, dans cette salle, qu'il faut le voir et l'entendre. Un peintre ferait un beau portrait s'il saisissait le caractère puissant de ce crâne dépouillé et poli, non par l'âge (M. Soury est jeune encore), mais par le travail de la pensée, de ces petits yeux perçants, de ces joues lourdes que la parole anime, de ce geste simple et paisible, de cette forme épaissie par une vie claustrale et qui révèle une vigueur de corps peu commune, détournée au profit du travail sédentaire et des spéculations intellectuelles. Je voudrais que le peintre mit toute la lumière sur ces mains un peu courtes, mais belles, qui après s'être plongées dans la prodigieuse substance blanche ou grise, s'ouvrent, pour la démonstration, comme afin de laisser échapper les vérités dont elles sont pleines. Ce serait vraiment une belle composition, et tout, jusqu'aux débris de cervelle et de cervelet répandus sur la table, prendrait un sens intellectuel, revêtirait cette noblesse que la science imprime à la nature. En dix ans de professorat, M. Jules Soury a pu faire la synthèse des travaux qui, depuis 1870, c'est-à-dire depuis la découverte de Fritsch et Hitzig, ont paru en Europe sur la théorie des localisations cérébrales. Il a publié la matière de ses leçons en un volume qui vient de paraître. ${ }^{65}$

Depuis 1881, les échanges épistolaires avec Haeckel se sont espacés et, dans ses rares lettres, Soury cherche surtout à obtenir des marques de reconnaissance ou simplement d'attention, souhaitant demeurer au premier rang des admirateurs et des propagateurs de la doctrine haeckelienne.

Le 10 octobre 1892, Soury adresse à Haeckel une longue lettre empreinte de pessimisme. Tout va mal en France, il y a une "dégénérescence de la raison »; tous les maitres à penser de la jeunesse sont redevenus déistes est spiritualistes, on croit désormais aux révélations des médiums et des âmes des morts... On assiste à la même réaction cléricale et obscurantiste qui fait pousser à Haeckel « ce grand cri d'alarme, Die Weltanschauung des neuen Kurses ${ }^{66}$. Soury cherche l'approbation de Haeckel dans son combat matérialiste ; mais Haeckel s'intéresse de plus en plus à la philosophie, et se tourne vers une propagande presque religieuse du monisme ${ }^{67}$.

Lorsqu'il envoie à Soury son livre Der Monismus als Band zwischen Religion und Wissenschaft ${ }^{68}$ , qui parle d'une «idée moniste de Dieu », de l'« unité de Dieu et du monde », de «Dieu et de la nature », d'un « Dieu-Nature », source inépuisable de jouissances esthétiques, enfin, de la nouvelle « trinité du monisme », le « beau », le « vrai » et le «bien », Soury est outré. Le 27 décembre 1892, il rédige une véritable « lettre de rupture » destinée à Haeckel, dans laquelle il le compare à Auguste Comte, « dont la ferveur religieuse fut un des symptômes de l'aliénation mentale»: «Je ne vous suis plus, je refuse énergiquement d'entrer dans l'Église nouvelle que vous dédiez à la Trinité du Monisme ! " ${ }^{69}$

La rupture semble consommée mais le lien se renoue lorsqu'en 1897, après un long silence, Soury remercie Haeckel, par une lettre anodine, pour l'envoi d'un livre et annonce son futur grand livre sur le système nerveux central. En effet, Soury tira de son enseignement la matière de nombreux articles parus dans des journaux médicaux ( L'Encéphale de Luys ; Les Archives de neurologie de Charcot) et de plusieurs livres, dont son opus magnum, Le Système nerveux central (1889), couronné par l'Académie de médecine et l'Académie des sciences ${ }^{70}$.

41 Cet ouvrage monumental (deux gros volumes in quarto, 1863 pages) a pour ambition de retracer une histoire anatomique et physiologique de l'intelligence, une "histoire naturelle de l'esprit humain $»^{71}$. Il débute par Alcméon de Crotone (500 avant J.C.) et se termine avec la théorie neuronale de Santiago Ramon y Cajal, à la pointe des recherches contemporaines les plus avancées. 327 pages sont dédiées à l'Antiquité, 33 au Moyen Âge, 269 à l'âge moderne, et 1150 à l'époque contemporaine. 67 pages de table des matières 
analytique, et 13 pages d'index nominum complètent cette somme. Le Système nerveux central est fondé sur un matérialisme et un évolutionnisme intransigeants :

La psychologie contemporaine est une psychologie sans âme. Confinée dans l'observation et dans l'expérience, la science, par définition, ne saurait rien et ne peut rien savoir de ce qui ne tombe pas sous les sens ou n'est pas l'objet d'expérience. C'est dire que loin d'affirmer ou de nier les croyances métaphysiques ou religieuses, la science les ignore. Là est la raison de son avancement, l'explication de sa puissance conquérante dans le présent, la promesse de son règne à venir. ${ }^{72}$

42 Ce qu'on appelle raison est ramené à des «instincts héréditaires » résultant « des variations utiles acquises mécaniquement au cours des longues luttes pour l'existence, ou, en d'autres termes, en vertu d'habitudes ancestrales devenues organiques par sélection naturelle ».

Descartes avait raison : tous les êtres vivants ne sont que des automates : son erreur a été de tirer l'homme de la foule innombrable de ses frères inférieurs. Inconscients ou conscients, les processus psychiques n'en sont pas moins toujours automatiques. La conscience n'ajoute rien, quand elle existe, à ces processus, pas plus que l'ombre au corps. ${ }^{73}$

43 À la fin de l'ouvrage, Soury souligne la continuité des mondes inorganique et organique :

L'unité suprême de la nature a sa plus haute expression dans l'unité de la science. Il n'y a pas deux mécaniques, une mécanique céleste et une mécanique cérébrale ; deux chimies, une chimie inorganique et une chimie organique ; deux physiologies, non plus que deux psychologies. Partout éclate, avec l'infinité de la causalité, la continuité des phénomènes naturels. ${ }^{74}$

En 1899, l'année même où il publie Le Système nerveux central, Soury s'engage dans la campagne antidreyfusarde: ses interventions visent à légitimer le nationalisme xénophobe et l'antisémitisme politique par un déterminisme biologique absolu ${ }^{75}$.

Mais une lettre à Haeckel datée du 2 janvier 1881 montre que l'antisémitisme de Soury ne date pas de la fin du siècle :

J'applaudis au mouvement antisémitique de votre nation. Mais il faut bien qu'on sache que le judaïsme n'est pas un fait religieux mais un fait de race, qu'un juif baptisé, germanisé, francisé, italianisé, etc., n'en reste pas moins toujours un juif, un sémite, dont la patrie véritable est dans la vallée du Jourdain. Le Sémite et les Indo-Européens constituent deux races humaines absolument hétérogènes, et, dans la lutte pour l'existence civile, raciale, économique, les Sémites, plus souples, plus capables de s'adapter aux conditions extérieures, l'emporteront à la fin, si l'on n'y prend garde, sur les Indo-européens. Cette fois, ce ne serait pas la meilleure, au moins moralement, mais la plus apte, qui triompherait, au grand dommage de la civilisation moderne. Bon courage donc, et sus aux Juifs !

Pour Soury, à l'inconscient cérébral ${ }^{76}$ s'ajoute un inconscient héréditaire, racial. Les « Aryens » et les « Sémites » seraient dotés d'une psychologie différente, car d'un cerveau différent: "Ces deux grands groupes ethniques réagissent tout autrement dans les mêmes circonstances parce que leur nature est hétérogène ». Les Juifs sont présentés comme une race ou peut-être même une espèce différente, inassimilable, toujours étrangère " en dépit de toutes les naturalisations, de toutes les conversions ", définie et emprisonnée par sa mémoire héréditaire, et menacée par la dégénérescence, «l'usure du système nerveux central». L'antisémitisme serait donc une réaction naturelle d'autodéfense des "Aryens" contre les Juifs, l'expression d'une "guerre des races». Soury donnera à L'Action française des articles virulents, contre «la Porcherie contemporaine » dirigée par «les francs-maçons, les Juifs et les huguenots », et pour la 
défense « de tout ce que nous aimons, la Terre de nos Morts, l'Église catholique, l'Armée de la France». Ces articles seront repris dans son dernier livre: Campagne nationaliste 1899-1901, dédié au général Mercier, dont il avait admiré la déposition contre Dreyfus au procès de Rennes en 1999, et auquel il aurait apporté personnellement une copie de son ouvrage $^{77}$.

Soury fonde son antisémitisme "scientifique » dans sa croyance aux lois d'airain de l'hérédité :

J'ai dédié cet ouvrage à la mémoire de mes parents, à ceux dont je ne suis, comme nous ne le sommes tous, que la continuité substantielle, la pensée et le verbe encore vivants avec leur cortège de gestes, d'habitudes et de réactions héréditaires, qui font que le mort tient le vif et que les caractères propres, ethniques et nationaux, nés de variations séculaires, qui différencient le Français de France de l'étranger, ne sont point des métaphores, mais des phénomènes aussi réels que la matière des éléments anatomiques de nos centres nerveux, les neurones, seuls éléments de notre corps qui, de la naissance à la mort de l'individu, persistent sans proliférer ni se renouveler jamais. Là est le témoignage irréfragable de l'hérédité psychologique. Là est le fondement de notre culte des morts et de la terre où ils ont vécu et souffert, de la religion de la patrie. ${ }^{78}$

Soury, ce célibataire pessimiste, a vécu avec sa mère jusqu'à l'âge de cinquante-trois ans. Après la mort de celle-ci, à l'âge de 86 ans, le 26 octobre 1895, terrassé, il se laisse presque mourir de faim ; « enseveli vivant », il déjeune d'un morceau de pain et d'une pomme sur un banc du jardin du Luxembourg ${ }^{79}$. Après l'effort presque surhumain de la rédaction du Système nerveux central, et les bouffées de haine de la Campagne nationaliste, Soury poursuit ses cours mais ne publie plus. Sa fin de vie est solitaire. Ses écrits antidreyfusards l'ont éloigné de la plupart de ses amis et collègues ${ }^{80}$. Par ailleurs, ses travaux sur le système nerveux ne lui paraissent plus aussi solides qu'il le croyait. Barrès rapporte en 1906 ces propos désabusés de Soury ${ }^{81}$ :

J'ai eu une grande contrariété. Les travaux espagnols exécutés grâce à des réactifs nouveaux m'ont prouvé que le lobe frontal, préfrontal ( ?) ne peut plus être le siège de l'intelligence comme je le croyais. Il n'a pas une richesse suffisante. C'est un espace déshérité, une plaine Saint-Denis. Ainsi ma construction s'écroule. Ignoramus et Ignorabimus. Ce n'est pas le temps de savoir, mais c'est toujours le temps de chercher. Mes matériaux me restent : nous avons les localisations, mais le siège de cette faculté de l'âme qu'on appelle l'intelligence, il reste à déterminer. C'était une cité de nuages. Un coup de vent est venu. Agnosticisme.

Des années après sa brouille avec Haeckel, le 17 février 1914, à l'occasion du 80 anniversaire du savant allemand, Soury lui envoie une longue lettre affectueuse, dans laquelle il rappelle leur longue amitiés ${ }^{82}$. Et le 26 juin, il lui adresse son grand livre, « que le disciple fidèle dépose aux pieds de son maître ».

Deux jours auparavant, le 24 juin 1914, l'archiduc François-Ferdinand avait été assassiné.

Le 17 juillet Soury demande à Haeckel s'il a reçu son livre.

Le 28 juillet, l'Autriche-Hongrie déclare la guerre à la Serbie et le 3 août, l'Allemagne déclare la guerre à la France. Le Temps du 13 octobre 1914 publie l'Appel aux Nations Civilisées (An die Kulturwelt), signé par 93 intellectuels et scientifiques allemands, dont Ernst Haeckel.

Soury meurt le 10 août 1915, à 73 ans, au 127, rue du Ranelagh, dans un oubli complet. Après des obsèques religieuses à Notre-Dame-de-Miséricorde d'Auteuil, il est porté au cimetière de Montparnasse, selon ses vœux, dans le corbillard des pauvres ${ }^{83}$. 
Dans son ouvrage sur Le Stupide XIX ${ }^{e}$ siècle (1922), Léon Daudet se souviendra de lui :

Je ne me rappelle pas sans attendrissement le papa Jules Soury, matérialiste convaincu et qui, cependant, par tradition, allait régulièrement entendre sa messe. À ses yeux la vie était exécrable, un deuil semé de deuils, et sans lendemain. Mais, pour franchir ce temps hideux (où les concierges et les domestiques lui apparaissaient ainsi que des fléaux en surcroît), il y avait la science et le laboratoire. Le papa Soury croyait dur comme fer, lui, l'incrédule, que la constitution du cerveau, considéré comme sécrétant la pensée à l'aide des neurones (mais où sont les neurones d'antan!), était aujourd'hui connue de façon définitive, immuable. Il se fâchait, si l'on émettait là-dessus quelque doute. Comme j'avais plaisir à le voir et à jouir de son humeur pittoresquement quinteuse, je lui accordais ce qu'il voulait ; et même que son énorme bouquin sur les fonctions du cerveau conserverait son actualité au-delà de trente ans. Il l'a déjà entièrement perdue. ${ }^{84}$

Haeckel survécut à Soury jusqu'en 1919.

\section{ANNEXES}

\section{Annexe 1. Lettre sans date (automne 1879)}

Cher Maître et ami,

Vous avez dû recevoir, ces jours-ci, un exemplaire cartonné du Règne des protistes ? L'avezvous reçu?

Voici maintenant quelques exemplaires de votre Psychologie cellulaire, que la librairie Germer Baillière vient de mettre en vente. Ce livre aura beaucoup plus de retentissement en France que le Règne des protistes, grâce à la grande publicité dont dispose la librairie Germer Baillière et Cie. J'ajoute que j'ai eu beaucoup plus à me louer de cette maison que de celle de M. Reinwald qui, en s'arrangeant pour publier votre livre sans rien payer, ou peu de chose, a mis en vente un livre mal disposé typographiquement, rempli de fautes d'impressions, que l'éditeur allemand a refusé de corriger, même dans un erratum !

J'ajoute encore que je n'ai eu que dix exemplaires (10 ex.) pour moi, et que la presse en a eu moins encore, si bien que, malgré tous mes efforts, le Règne des protistes passe presque inaperçu, et cela par l'avarice des éditeurs allemand et français de votre livre.

$\mathrm{Au}$ contraire, la Psychologie cellulaire aura un grand retentissement dans toute la France.

Dans une courte préface, j'ai présenté le mieux que j'ai pu au public français votre profonde hypothèse de la périgenèse des plastidules. Voilà le fondement véritable de toute psychologie de l'avenir, laquelle devra être une " psychologie sans âme ", - cette abstraction ne faisant qu'embrouiller et compliquer le problème biologique dont il s'agit, et perpétuer des habitudes de langage dont profitent nos plus dangereux ennemis, les spirites de Londres et de Leipzig, de Russie et d'Amérique. 
La sensibilité, c'est-à-dire la propriété de réagir contre les impressions du milieu ambiant, peut bien être une propriété générale de la matière organique et inorganique, s'il est encore permis de conserver cette distinction : outre que cette sensibilité doit être inconsciente chez les minéraux et chez les protistes les plus rudimentaires, tels que les monères, le mot « âme » n'éclaire en rien la nature du processus de la sensation et prête à toute sorte d'erreurs.

Puisque la vie commence avec l'apparition de certaines conditions, il est naturel d'admettre que les propriétés vitales, servant à définir la vie, - telles que nutrition, reproduction, sensibilité et mouvement volontaire - commencent également avec les combinaisons albuminoïdes ; il est donc tout à fait hypothétique de transporter ces propriétés vitales aux molécules minérales et aux atomes chimiques.

Au fond, je sais, cher Maître et ami, que je suis tout à fait d'accord avec vous. En France, on serait heureux de vous voir écrire une Psychologie cellulaire complète, formant en quelque sorte un pendant au grand livre de Virchow. Quoi que vous écriviez, d'ailleurs, vous avez parmi nous d'enthousiastes disciples, votre philosophie biologique étant le couronnement naturel de la conception mécanique du monde de votre Kant et de notre Laplace.

Tenez-moi, je vous prie, au courant de vos belles recherches ; envoyez-moi ce que vous publiez, livres ou articles, afin que je continue d'entretenir de vos idées et de vos découvertes le public français. Vous écrivez dans le Kosmos, dans la D. Rundschau, dans les journaux savants d'Iéna, tous recueils que je ne puis pas toujours lire assidûment ; veuillez donc m'envoyer des tirages à part de vos articles.

Veuillez croire, je vous prie, cher ami, a mes sentiments d'affectueux dévouement et d'ardente sympathie.

Jules Soury

Paris, 52 boulev. St. Germain

\section{Annexe 2. Lettre du 10 octobre 1892}

Paris, 21 rue Gay Lussac

le 10 oct. 1892

Cher maître et ami,

je m'empresse de vous adresser mes plus vifs remerciements pour l'envoi de vos dernières publications, pour la $3^{\circ}$ édition de votre grand livre, l'Anthropogénie, si profondément remanié et toujours si génial, mais aussi pour les deux autres opuscules qui l'accompagnent.

Je vois que vous vous préparez à publier une édition, tout à fait nouvelle, de votre Protistenreich. Je serai heureux si, comme vous voulez bien me le faire espérer, l'honneur m'est réservé de traduire encore ce livre. S'il vous plaît que j'y mette une introduction, celle-ci aura des proportions beaucoup plus restreintes que l'ancienne.

Reste la question de l'éditeur. Je ne connais pas les successeurs de feu M. Reinwald. Mais peut-être êtes-vous en rapport avec eux et savez-vous leurs intentions quant à cette nouvelle édition? 
Naturellement, il y a plus d'un éditeur pour Ernst Haeckel à Paris, et, quoi qu'il arrive, vous êtes de ceux qui sont toujours sûrs d'être lus, en français comme en allemand.

Je vous envoie par courrier mes Fonctions du cerveau, et je sollicite toute votre amicale indulgence pour l'œuvre et pour l'auteur. Le Professeur Hitzig de Halle a bien voulu écrire deux articles favorables, pour la $1^{\text {re }}$ et la $2^{\mathrm{e}}$ édition de ce livre, et le professeur H. Munk, le physiologiste de Berlin, s'en réfère à mon livre, pour l'histoire de la question, dans sa dernière communication à l'Académie des Sciences de Berlin, intitulée Über die Gefühlsphären des Grosshirns. Mais c'est à votre estime, à votre approbation que je tiens par dessus tout, en souvenir de ma vieille et toujours vivace admiration pour vous, pour le savant et pour le philosophe.

Nous assistons, en France à la même réaction cléricale et obscurantiste qui vous fait pousser ce grand cri d'alarme, Die Weltanschauung des neuen Kursus. Sous prétexte de réconciliation et d'union, le gouvernement de la République française accueille depuis quelque temps, et favorise de toutes les manières, les pires ennemis de la libre pensée et de la science, les catholiques, les chrétiens de toute secte et les Juifs. La France ne périra que par Rome ; la papauté ne disparaîtra du monde qu'en entraînant après elle la France dans l'abîme.

La Weltanschauung de nos écoles - depuis l'école primaire jusqu'aux Universités, est encore et toujours celle des livres hébreux, des Pères de l'Église et des scholastiques du moyen âge. Tous les maîtres de la jeunesse que l'on forme dans les Écoles normales sont redevenus déistes et spiritualistes comme on l'était en France il y a cinquante ans. La conception mécanique du monde n'existe plus que dans quelques intelligences, rari nantes in gurgite vasto ${ }^{85}$. Les socialistes sont ignorants, et les gens d'étude, la jeunesse comme les professeurs, se convertissent de plus en plus à une sorte de religion mystique, de superstition savante, qui semble un retour atavique aux idées des sauvages sur le monde ; les grands prêtres de cette religion s'appellent Wallace, Krooks, Zöllner, Ch. Richet, le professeur de physiologie de la Faculté de médecine de Paris !

On fait aujourd'hui profession de dédaigner la science et l'on exalte la foi, - la foi aux choses de l'au-delà, aux révélations des médiums et des âmes des morts. Les hallucinations télépathiques, les suggestions à distance, le transfert des forces nerveuses, voilà la matière des livres et des conversations chez les Européens les plus instruits de cette fin de siècle. En effet en Allemagne, et non pas seulement en France, en Russie, en Angleterre surtout, maintenant aussi en Italie, même dégénérescence, à de degrés divers, de la vieille raison humaine. L'occultisme, c'est à dire le spiritisme et le spiritualisme, se propage et sévit, à la manière d'une épidémie, sur toute l'Europe.

Quelle sera la fin de tout cela ? On en reviendra sans doute à l'acception du monde d'un Démocrite, d'un Galilée, d'un Laplace et d'un Darwin ; mais le mal qui nous envahit me paraît devoir s'appesantir pour ( ?) des années sur cet Occident, qu'on pouvait croire à l'abri de pareils fléaux.

Songez que Bismarck lui-même a été à Canossa. Le pape, comme vous l'avez écrit, a triomphé du chancelier de fer. Que pouvons-nous, nous autres qu'on hait et qu'on persécute de toutes les manières, pour sauver ces millions d'intelligences héréditairement faussées, livrées en proie, dès la première enfance, aux prêtres, aux maîtres confessionnels, enfin à la caserne? 
Nous avions cru les conquérir à nos idées, à la science, à la philosophie. Nous avons fait un beau rêve. Nous n'avions de prise que sur les intelligences; ce qu'il y a de profond et d'indestructible dans la nature humaine, les sentiments et les instincts, sont demeurés les mêmes ; et le premier faiseur de pluie, le premier sorcier venu n'a eu qu'à paraître : tous ces faux convertis sont revenus aux façons de sentir et de penser ancestrales, à la foi aux revenants, à l'invisible, au surnaturel.

Certes, la science s'accroîtra et persistera aussi longtemps que l'intelligence humaine sur cette planète, mais elle sera de plus en plus la chose d'une aristocratie spirituelle, suspecte aux foules, et souvent dénoncée aux pouvoirs publics, aux Parlements, aux Églises, aux Caprivi et aux Zedlitz, comme une puissance funeste aux États et dangereuse pour l'esprit public.

Pardonnez-moi ces réflexions chagrines que m'a inspirées votre bel article, Die Weltanschauung des neuen Kursus, et croyez-moi toujours, je vous prie, tout à vous de cœur et d'esprit

Jules Soury

J'envoie par le même courrier un exemplaire de mon livre à votre très distingué disciple, M. le $\mathrm{D}^{\mathrm{r}}$ Max Verworn. Présentez-lui mes excuses pour ne pas avoir mis de dédicace : je n'avais plus d'exemplaire, et j'ai dû écrire à l'éditeur. Veuillez avoir aussi la bonté de transmettre l'erratum ci-joint.

\section{Annexe 3. Lettre du 27 décembre 1892}

Paris, 21 rue Gay-Lussac

le 27 décembre 1892

Très honoré et illustre ami,

J'ai tenu, avant de vous remercier, à lire la dernière publication que vous m'avez fait l'honneur de m'envoyer, Der Monismus, afin de vous faire connaître mon sentiment. Vous êtes un trop grand esprit, et le sort nous a faits [sic] citoyens de mondes trop différents et trop éloignés, pour que je ne vous parle pas avec toute la liberté dont useraient les habitants de deux planètes, s'ils pouvaient converser comme nous le faisons.

Ce n'est pas, je vous l'avoue, sans une secrète inquiétude que j'ai lu, sur la couverture de votre livre, les mots de " profession de foi » et de « religion ». Jugez de ma surprise, quand je vous ai entendu parler d'une « idée moniste de Dieu », de l'« unité de Dieu et du monde », de "Dieu » et de la "nature », d'un « Dieu-Nature », source inépuisable de jouissances esthétiques, enfin, de la nouvelle « Trinité du monisme », le « beau », le " vrai » et le « bien », Trinité à laquelle le vingtième siècle doit élever des autels !

Je serai franc et loyal comme vous l'avez toujours été, dans vos discours comme dans vos livres : je ne puis vous suivre sur ce terrain-là. Jusqu'à ce manifeste religieux, j'ai été votre disciple, votre admirateur enthousiaste. Mais ici je m'arrête, je ne vous suis plus, je refuse énergiquement d'entrer dans l'Église nouvelle que vous dédiez à la Trinité du Monisme !

Tant que vous avez parlé de la Nature et de ses lois, je vous ai compris. D'un Dieu et d'une religion, je ne sais rien et ne veux rien savoir. Le savant et le philosophe ont désappris cette langue pour toujours. Tant pis si les militaires, les magistrats, les prêtres, les rois le 
regrettent, mais quand se lève l'aurore de la Révolution sociale, quand la science n'a plus rien à redouter des princes ni des tribunaux ecclésiastiques, venir nous parler de « Dieu », d' " âme », de " religion ", d' « éthique » et d' " esthétique », c'est laisser paraître qu'on est victime d'une tare héréditaire, d'une survivance des âges de foi, d'une régression atavique de l'esprit.

En France, votre Discours n'aurait pu être prononcé dans une réunion de naturalistes et de médecins. Individuellement, ces savants peuvent partager vos idées, ou même celle d'une Église quelconque, mais parler en public de Dieu, d'âme ou de religion, voilà ce que personne n'oserait faire, aujourd'hui, à Paris. C'est un cas de respect humain. Les journaux où l'on imprime encore ces mots-là, sont des torchons de sacristie, lus par quelques cerveaux séniles, en démence.

Pourquoi voulez-vous appeler la Nature « Dieu », et « âme » les propriétés psychiques de la matière vivante ? Est-ce donc uniquement pour faire plaisir aux bourgeois ignorants, mais bien pensants, qui, en tous pays, veulent une « religion » pour leurs femmes, leurs enfants et leurs domestiques? Loin d'imaginer, comme un apologiste chrétien, des rapports et des liens entre la science et la foi, le savant laisse les croyants à leurs autels et ne sait rien d'une religion. Le savant conçoit, au point de vue historique, la nécessité, et partant la légitimité, des diverses religions : elles correspondent à des états définis que l'esprit humain a dû traverser ; le savant n'inquiète pas les croyants ; il ne les raille pas ; il les étudie curieusement ou les ignore. Mais, quant à créer une religion nouvelle, sous le nom de monisme, avec ou sans Trinité, on ne l'en aurait jamais cru capable, du moins depuis Auguste Comte, dont la ferveur religieuse fut un des symptômes de l'aliénation mentale.

La conception moniste de l'univers n'implique nullement une religion, c'est à dire une mythologie dans laquelle les quelques lois connues de l'univers et les qualités intellectuelles et morales d'un mammifère sont hypostasiées. Le beau, le vrai, le bien ! Je ne veux pas vous rappeler que ces mots sont le titre d'un livre célèbre du père de la philosophie éclectique en France, de Victor Cousin, et qu'on se couvrirait de ridicule chez nous si l'on osait avouer qu'on a lu ce livre-là.

Mais, d'une manière générale, qu'est-ce que le bien, le vrai, et le beau, sinon de purs concepts, variant, au cours des âges, avec les races humaines, avec les latitudes, avec les états différents de civilisation? Or un concept ne correspond à rien d'objectif. Dans la nature, il n'y a ni bien ni mal, ni vérité ni erreur, ni beauté ni laideur. Ces fantômes ne hantent que notre esprit ; ils s'évanouiront avec le dernier homme.

N'allez pas croire surtout que je vous parle ainsi au nom d'une philosophie adverse quelconque. L'athéisme et le matérialisme sont aussi vains que le déisme et le spiritualisme. Le savant digne de ce nom, ou simplement le philosophe instruit, ne perd plus son temps à soutenir ou combattre des thèses d'École. Nous ignorons et nous ignorerons toujours l'étoffe dont est fait ce monde. Atomes ou monades? On ne le saura jamais. En dehors de nos sensations et de nos représentations, nous ne connaissons rien ; l'univers, tel qu'on le construit en mécanique, en physique et en chimie, n'est qu'un monceau d'inductions. Pour expliquer la vie, la sensation et la pensée, c'est à dire pour réduire ces processus à des considérations de chimie, de physique et de mécanique, nous avons recours aux mêmes principes, aux principes de ces sciences. 
Au fond de tout, qu'il s'agisse de sociologie, de psychologie, de physiologie ou d'astronomie, il n'y a pour nous, hommes, qu'un problème de mécanique. S'il y a autre chose dans l'univers que des mécanismes, nous n'en savons rien : nous devons donc nous abstenir d'hypothèses invérifiables.

Tout ce qui est au-delà de l'observation et de l'expérimentation, est pour nous l'inconnu et l'inconnaissable. Or, là où règnent les lois d'airain de la mécanique et du déterminisme universel, il n'y a pas de dieu, il n'y a pas d'âme, il n'y a pas de bien, de vérité, de beauté : il n'y a pas de religion.

En pensant ainsi, et je n'ai guère varié depuis que je réfléchis, je ne suis le sectateur d'aucune philosophie. Je me résigne humblement à rester dans les limites de l'observation et de l'expérience.

Ces doctrines, vous les avez trouvées exposées, s'il vous en souvient, dans les préfaces que j'ai mises en tête des traductions que j'ai faites de quelques-uns de vos ouvrages ; je croyais qu'elles étaient en harmonie avec vos propres pensées. Je me serai trompé.

Vous invoquez souvent les grands noms de Démocrite et de Darwin : tenez pour assuré que ni l'un ni l'autre de ces génies n'aurait mêlé la religion à la science.

Pardonnez-moi, je vous prie, cette longue critique de vos idées nouvelles, et croyez toujours à ma vieille amitié, à ma sympathie, à ma sincère admiration.

$\mathrm{D}^{\mathrm{r}}$ Jules Soury

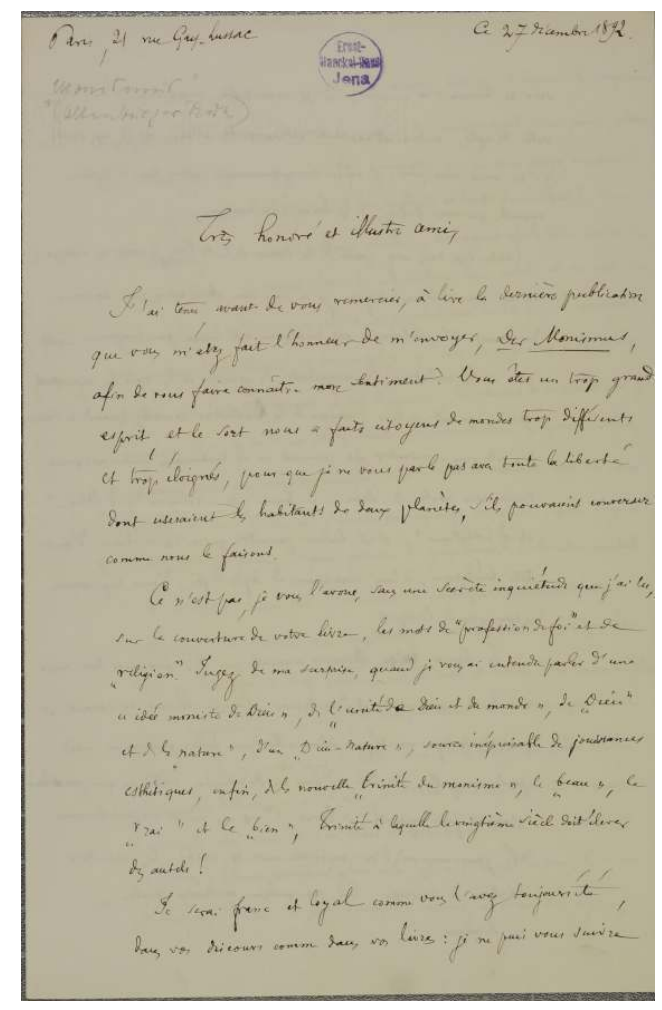



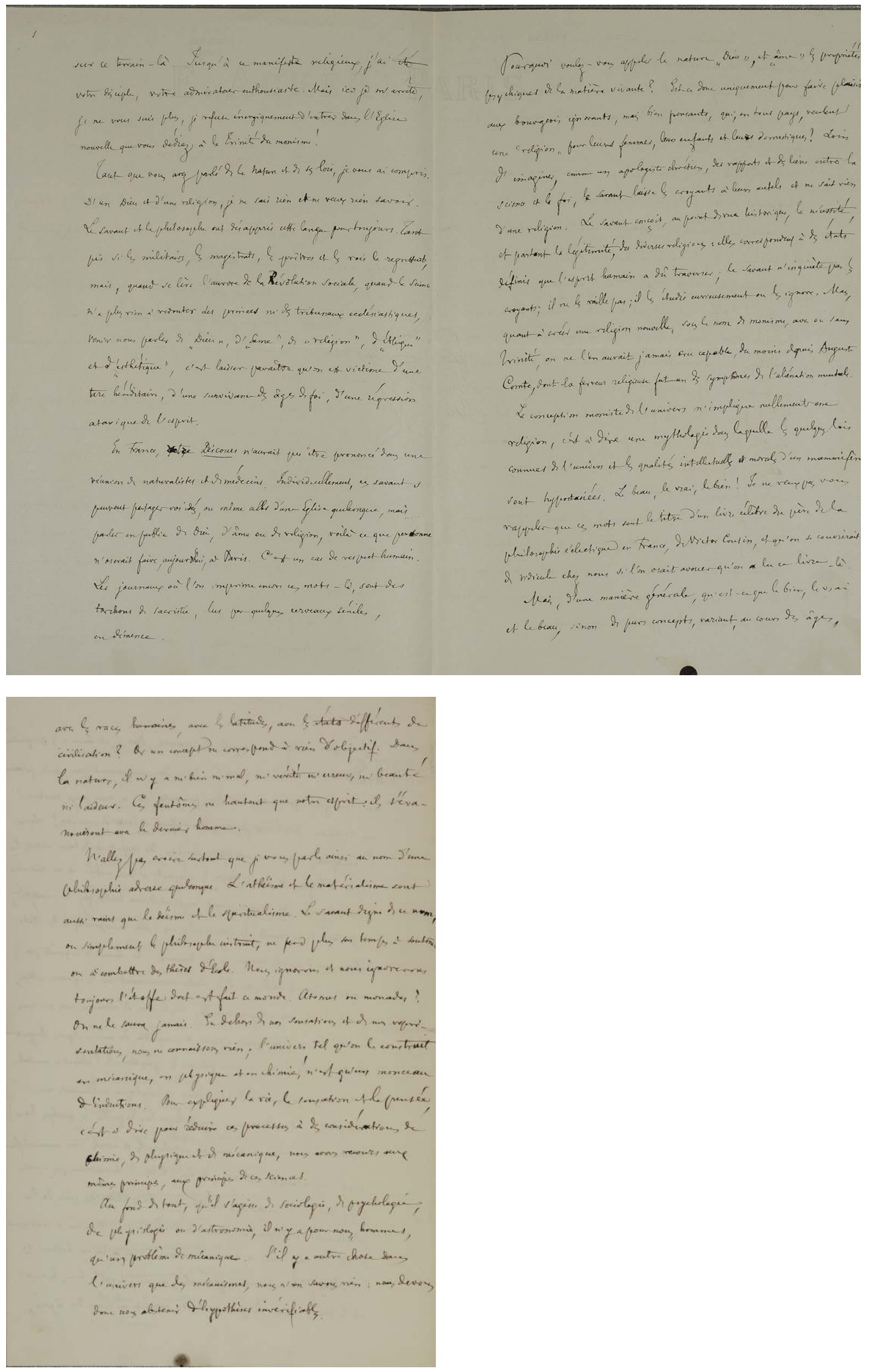


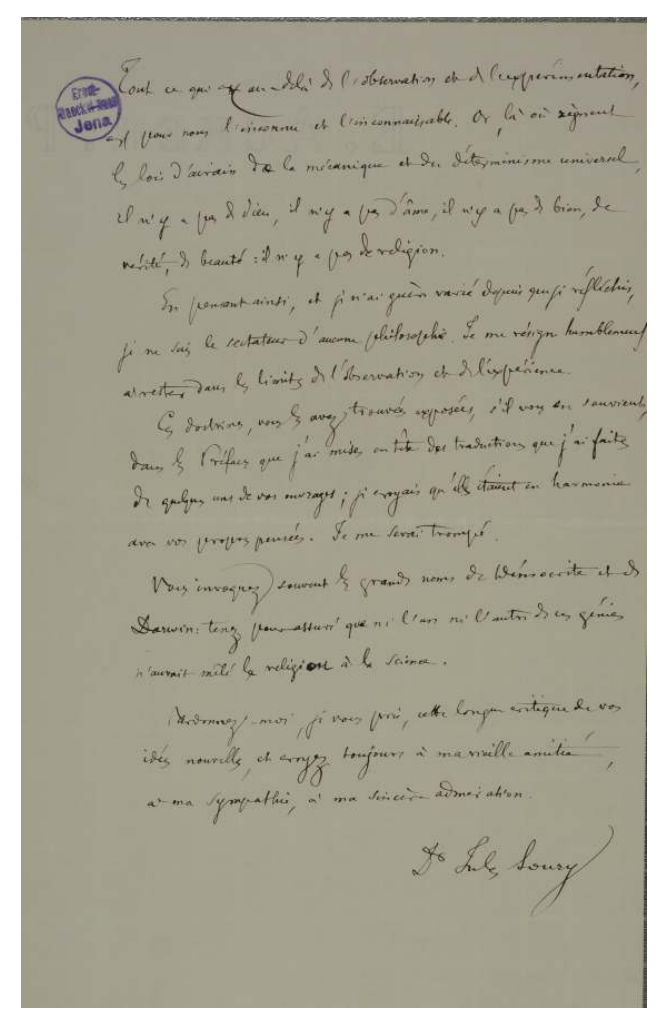

\section{Annexe 4. Lettre du 17 février 1914 (3 pages sur papier en tête de l'École des Hautes Études)}

Paris, le 17 février 1914

127, rue du Ranelagh

Très honoré Maître,

cher et grand ami,

J'apprends aujourd'hui seulement qu'on vient de célébrer, à léna, votre quatre-vingtième anniversaire.

Que n'étais-je auprès de vous! J'aurais aimé à témoigner publiquement, comme je l'ai fait toute ma vie, de ma profonde admiration pour votre science et pour votre conscience, pour votre œuvre géniale ! La bonté de votre cœur, la loyauté de votre nature peuvent seules égaler la sereine grandeur de votre caractère.

Je parle ainsi, parce que j'ai pu personnellement vous juger.

Vous rappelez-vous votre voyage à Paris, d'il y a tant d'années, au lendemain de la Guerre, déclarée à l'Allemagne par la France de l'Empire et par l'Église romaine? J'eus l'honneur de parler à vos côtés au Banquet que quelques savants français avaient offert au savant naturaliste d'Iéna.

Et mes traductions de quelques-uns de vos ouvrages, - la Psychologie cellulaire, les Preuves du Transformisme, le Règne des Protistes, vous en souvenez-vous encore? 
Quelles préfaces j'écrivis alors, dans mon enthousiasme et ma foi ! Et cette foi en la Théorie de l'Évolution est demeurée entière, et mon enthousiasme pour le maître d'Iéna a toujours grandi.

Nous avions déjà contre nous, en France, le plus grand nombre des Universitaires, des catholiques, des protestants, des Juifs, tous gens très avertis, et qui d'avance sentaient bien que la patrie de Lamarck, le pays qui continua d'ignorer le fondateur du transformisme et de méconnaître Charles Darwin, ne serait jamais de cœur ni d'esprit avec Haeckel. D'autres de vos grands livres en traduction française ont eu plus de succès, grâce à l'éditeur Reinwald, qui ne vous a pas trahi.

Malgré tout, et quoique le transformisme ait eu ses Charles Martins et ses Alfred Giard, la Théorie de l'Évolution des êtres organisés répugne aux esprits frivoles, ignorants ou sectaires, qui forment l'immense majorité des Français. Jamais le principe fondamental biogénétique ne pénétrera dans ces esprits, simplices et clairs comme celui de Voltaire. En dehors de l'élaboration des Constitutions politiques et des révolutions de la mode, les Français ne sauraient s'intéresser à rien. Les questions de l'origine et de la nature des choses, de l'univers et de la vie, les laissent proprement indifférents, n'étant point susceptibles de solutions positives et pratiques. En France, les animaux continuent à n'avoir point de fonctions psychiques, non plus que les végétaux. Un Leibnitz serait aussi peu compris qu'un Kant. Notre Goethe s'est appelé Victor Hugo.

Il n'importe, après tout. Chaque race, comme chaque individu, est ce qu'elle doit être, et ne saurait être autre. Ne jugeons pas ; constatons. Moi-même, je ne suis point, comme vous, cher et grand ami, pacifiste, et, quoique moniste, j'abhorre la Religion de l'Humanité, votre religion de l'Avenir, die Religion der Zukunft, avec ses symboles du Beau, du Vrai et du Bien. À cet égard, mais seulement à cet égard, je suis aussi peu Haeckelien que possible. Je demeure, comme les grands penseurs hellènes du VI siècle, hylozoïste et athée.

J'ai continué à vous lire, à exposer vos doctrines dans mon enseignement et dans mes livres, à exalter en Haeckel l'homme et le savant, car je vous honore et vous aime autant que je vous admire.

Recevez donc, je vous en prie, en ce jour, qui est celui de l'Anniversaire de vos quatrevingts [sic] ans, l'hommage d'un cœur fidèle, d'un vieillard de soixante-douze ans, dont la longue vie vous doit ses meilleures heures, des heures de travail et de méditation.

Jules Soury

$\mathrm{D}^{\mathrm{r}}$. de l'Université de Paris, Directeur d'Études à l'École pratique des Hautes Études à la Sorbonne, lauréat de l'Académie des sciences et de l'Académie de médecine. 

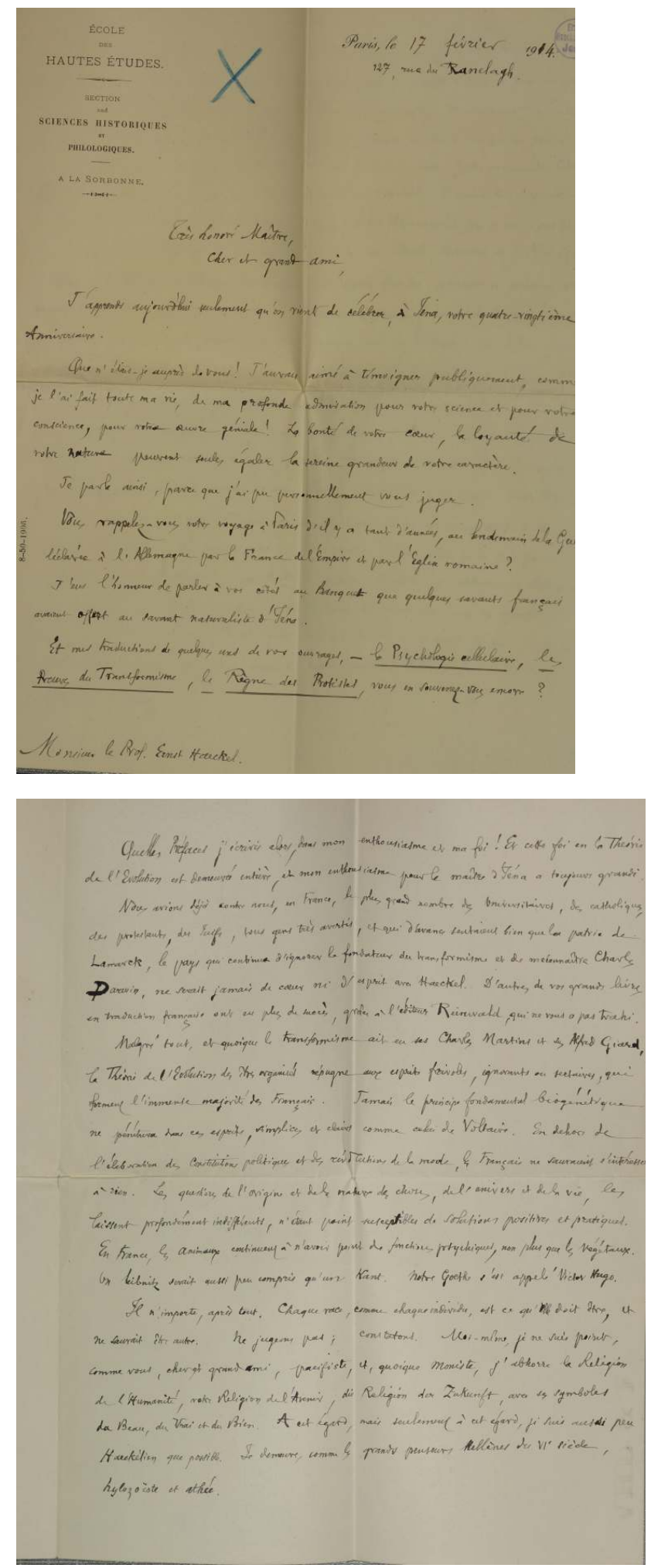


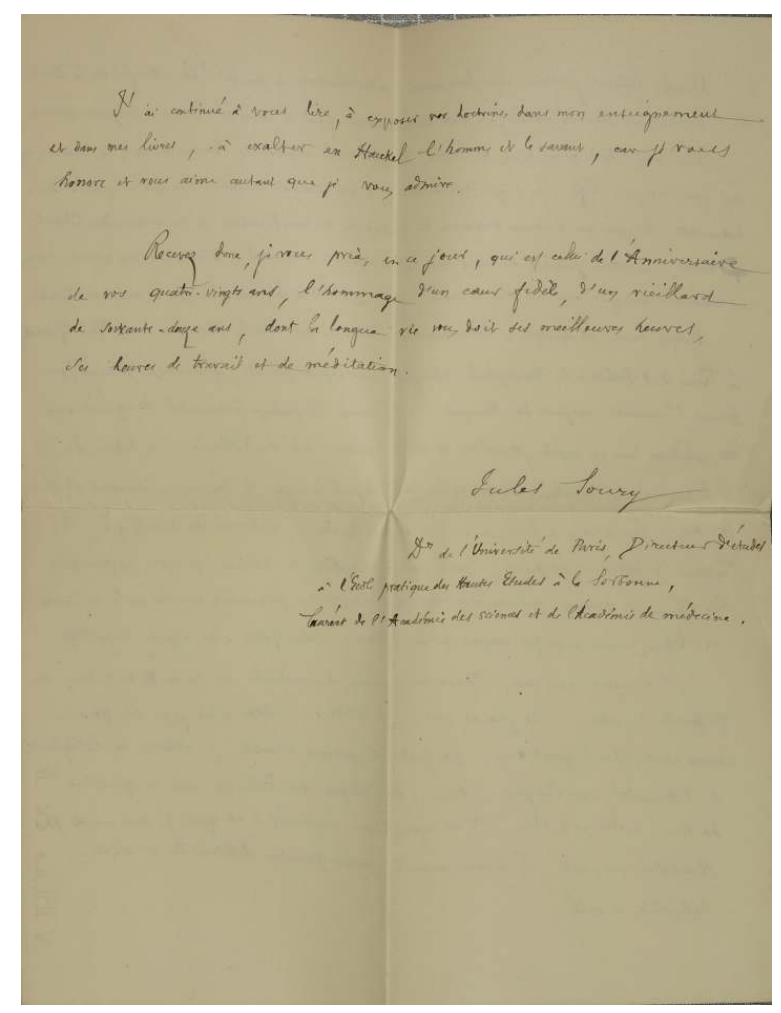

\section{NOTES}

1. Sur Ernst Haeckel et son œuvre, voir : Stephen Jay Gould, Ontogeny and phylogeny, Cambridge, Mass., Harvard University Press, 1977 ; Erika Krauße, Ernst Haeckel, Leipzig, Teubner, 1987 ; Mario Di Gregorio, From here to eternity. Ernst Haeckel and scientific faith. Göttingen, Vandenhook \& Ruprecht, 2005; Robert J. Richards, The Tragic Sense of Life, Chicago, University of Chicago Press, 2009; Laura Bossi, «Ernst Haeckel. De la Naturphilosophie romantique à la révolution darwinienne », Romantisme et Révolution III : Achèvement et dépassement, Paris, Gallimard, 2009.

2. Jacques Roger, «Darwin, Haeckel et les Français », Yvette Conry dir., De Darwin au Darwinisme : science et idéologie. Congrès international pour le centenaire de la mort de Darwin, Paris, Vrin, 1983, p. 149-165, repris dans Jacques Roger, Pour une histoire des sciences à part entière, Paris, Albin Michel, 1995, p. 372-393. Voir notamment p. 386.

3. Nous en donnons ici la liste: Histoire de la création des êtres organisés d'après les lois naturelles, trad. Charles-Jean-Marie Letourneau, avec une introduction biographique par Charles Martins, Paris, Reinwald, 1874 ; Anthropogénie, ou Histoire de l'évolution humaine, trad. Charles-Jean-Marie Letourneau, Paris, Reinwald, 1877 ; Les Preuves du transformisme, trad. Jules Soury, Paris, GermerBaillière, 1879 ; Le Règne des protistes, trad. Jules Soury, Paris, Reinwald, 1879 ; Essais de psychologie cellulaire, trad. Jules Soury, Paris, Germer-Baillière, 1880 ; Lettres d'un voyageur dans l'Inde, trad. Charles-Jean-Marie Letourneau, Paris, Reinwald, 1883 ; Le Monisme : profession de foi d'un naturaliste , trad. Georges Vacher de Lapouge, Paris, Reinwald-Schleicher frères, 1897 ; État actuel de nos connaissances sur l'origine de l'homme, trad. Léon Laloy, Paris, Reinwald-Schleicher frères, 1900 ; Les Énigmes de l'Univers, trad. Camille Bos, Paris, Reinwald-Schleicher frères, 1902 ; Religion et évolution. Trois conférences faites à Berlin le 14, 16 et 19 avril 1906, trad. Camille Bos, Paris, ReinwaldSchleicher frères, 1907 ; Les Merveilles de la vie, études de philosophie biologique, sans indication du traducteur, Paris, Reinwald-Schleicher frères, 1907. 
4. Die Radiolarien (Rhizopoda radiaria): eine Monographie, Berlin, Georg Reimer, 1862-1888 ; Generelle Morphologie, op.cit., 1866 ; Die Kalkschwämme I. Biologie der Kalkschwämme (Calcispongien oder Grantien): Eine Monographie in zwei Bänden Text und einem Atlas mit 60 Tafelnabbildungen..., Berlin, Georg Reimer, 1872.

5. Voir Linda Clark, Social Darwinism in France, Tuscaloosa, The University of Alabama Press, 1984 ; Joy Harvey, «L'évolution transformée: positivistes et matérialistes dans la Société d'Anthropologie de Paris du Second Empire à la III République », Britta Rupp-Eisenreich dir., Histoires de l'anthropologie (XVI $e^{e}$ XIX ${ }^{e}$ siècles), Paris, Klincksieck, 1994 ; Piet Desmet, La Linguistique naturaliste in France (1867-1922). Nature, origine et évolution du langage, Leuven-Paris, Peeters, 1996 ; Jacqueline Lalouette, La Libre Pensée en France 1848-1940, Paris, Albin Michel, 1997 ; Jean-Claude Wartelle, «La Société d'Anthropologie de Paris de 1859 à 1920 », Revue d'Histoire des Sciences Humaines, 2004, n 1 (10), p. 125-171; Claude Blanckaert, "L'impartiale Critique de la raison mûre... Évolution, libre pensée et monisme à la française", Jahrbuch für Europäische Wissenschaftskultur, 2007, $\mathrm{n}^{\circ}$ 3, p. 135-164.

6. C'est la définition de Marcel Gauchet, dans L'Inconscient cérébral, Paris, Seuil, 1992, p. 123.

7. Voir son autobiographie, dans Campagne nationaliste, 1899-1901. Autobiographie, Paris, Imprimerie de L. Maretheux, 1902; voir aussi André Rouveyre, Souvenirs de mon commerce: Gourmont Apollinaire - Moreas - Soury, Paris, G. Crès, 1921 ; Jean-Baptiste Mousson-Lanauze, «Jules Soury. Communication présentée à la Société d'Histoire de la Médecine, séance du 4 Juin 1927 », Paris Médical, 1927, $\mathrm{n}^{\circ}$ 66, p. 30-35 ; Camille Vettard, «Le drame de Jules Soury », La Revue Universelle 1939, n 76, p. 257-272 ; Pierre Huard, Marie-José Imbault-Huart, «Jules Soury (1842-1915)», Revue d'Histoire des Sciences, 1970, n² 23, p. 155-164 ; Toby Gelfand, « From religious to bio-medical anti-semitism : the career of Jules Soury ", Anne LaBerge et Mordechai Feingold dir., French medical culture in the nineteenth century, Amsterdam et Atlanta, Rodopi, 1994, p. 248-279; Zeev Sternhell, « Jules Soury, un raciste français ", Les Collections de l'Histoire, 1998, n 3, p. 12.

8. Anatomiste et neuropsychiatre d'abord à la Salpêtrière, puis à la Charité, fondateur de la revue L'Encéphale, il a décrit le noyau subthalamique (noyau de Luys) qui est aujourd'hui l'une des cibles de la stimulation cérébrale profonde pour le traitement de la maladie de Parkinson. Luys est représenté (à droite, à côté de son chef de clinique Gérard Encausse) dans le célèbre tableau Les Fascinés de la Charité (1889) de Georges Moreau de Tours (1848-1901), aujourd'hui au Musée de Reims.

9. Aliéniste élève d'Esquirol, depuis 1865 médecin en chef à Bicêtre du service pour enfants « disgraciés par la nature, mal nés, nés pauvres d'esprit », fondateur avec Jean-Pierre Falret de la maison de santé de Vanves ; en 1867, il succède à Pierre Falret à la Salpêtrière, où il s'intéressera de plus en plus à l'hypnotisme et à la suggestion.

10. Jules Soury, Essais de critique religieuse, Paris, E. Leroux, 1878.

11. Id., Jésus et les Évangiles, Paris, Charpentier, $1878 ; 3^{\mathrm{e}}$ éd. en 1898 , avec le titre : Jésus et la religion d'Israël.

12. David Friedrich Strauß, Das Leben Jesu, kritisch bearbeitet (1835), traduit en français par Émile Littré entre 1839 (t. 1) et 1853 (t. 2).

13. Robert Priest, "After the God and the man, the patient: Jules Soury's psychopathology of Jesus and the boundaries of the science of religions in the early Third Republic", French History, 2017, n -27(4), p. 535-556.

14. Voir note 3.

15. À sa création en 1868, l'EPHE avait pour mission d'introduire dans le monde universitaire un mode de formation fondé sur les séminaires et le travail en laboratoire. Elle comprenait alors quatre sections : Mathématiques, Physique et chimie, Sciences naturelles et physiologie, Sciences historiques et philologiques. Une $\mathrm{V}^{\mathrm{e}}$ section de Sciences religieuses y est adjointe en 1886.

16. Le Système nerveux central, structures et fonctions, histoire critique des théories et des doctrines, Paris, G. Carré et C. Naud, 1899. 
17. Dans l'ouvrage de référence de Haymaker et Schiller, il figure comme neurohistorian; c'est le seul «fondateur » de la neurologie non médecin. Webb Haymaker et Francis Schiller dir., The Founders of Neurology, $2^{\mathrm{e}}$ édition, Springfield, Ill., Charles C. Thomas, 1970, p. 573-576. Sur l'oeuvre d'historien de la neurologie de Soury, voir aussi : Toby Gelfand, "Jules Soury, Le système nerveux central (Paris, 1899) ", Journal d'histoire des neurosciences, 1999, n 8(3), p. 235-247; et Lazaros C. Triarhou, "Jules-Auguste Soury (1842-1915): A Centennial Call to Mind”, European Neurology, 2016, $\mathrm{n}^{\circ}$ 75(1-2), p. 12-25.

18. Paul Bourget, Le Disciple, Paris, Alphonse Lemerre, 1889 ; réédition par A. Compagnon, Paris, Le livre de Poche, 2010. La ressemblance a été notée par Toby Gelfand dans son article de 1994, "From religious to bio-medical anti-semitism : The career of Jules Soury", op. cit.

19. Camille Vettard, "Le fournisseur d'idées de Barrès, Jules Soury », Du côté de chez... Valéry, Péguy et Romain Rolland, Proust, Gide, Barrès et Soury, Sartre, Benda, Nietzsche, Paris, Éditions de la Tête noire, 1946, p. 61-85 ; Zeev Sternhell, Maurice Barrès et le nationalisme français, Paris, Pluriel, coll. «Pluriel », 2016 ( $1^{\text {re }}$ éd. 1972, Armand Colin). Les Archives Barrès (BN) conservent une centaine de lettres de Soury à Barrès, ainsi que de nombreux livres dédicacés de Soury.

20. Voir son autobiographie, dans Campagne nationaliste, op. cit., 1902 ; voir aussi Jean Serc, « Un clérical athée. M. Jules Soury », Mercure de France, 1905, n 56, p. 215-230.

21. Voir note 3.

22. Histoire de la création..., p. 17, 60-67 et passim.

23. Haeckel applique le darwinisme à l'homme dès sa conférence de Stettin, en 1863, bien avant la publication de The Descent of Man par Darwin en 1871.

24. Jacques Roger résume l'entreprise de Haeckel par la formule suivante: Haeckel ramène l'hérédité à la reproduction, et la variation à l'adaptation: Pour une histoire des sciences à part entière, op. cit., p. 377.

25. Pour Haeckel l'embryologie est fondamentale car elle rend vraisemblable la phylogenèse et permet d'en reconstituer les métamorphoses. Voir Jacques Roger, op. cit., p. 379.

26. 300 pages sur les 600 de l'Histoire de la création... sont dédiées à cette « généalogie des formes vivantes ", première tentative de classification phylogénétique du vivant.

27. Sur l'histoire du monisme, voir Rudolf Eisler, Geschichte des Monismus, Leipzig, Alfred Kröner Verlag, 1910 ; Horst Groschopp, Dissidenten : Freidenkerei und Kultur in Deutschland, Berlin, Dietz Verlag, 1997 ; Todd H. Weir éd., Monism. Science, philosophy, religion, and the history of a worldwiew, New York, Palgrave Macmillan, 2012. Sur le monisme haeckelien, voir notamment Paul Ziche dir., Monismus um 1900. Wissenschaftskultur und Weltanschauung. Ernst-Haeckel-Haus-Studien, Band 4, Berlin, Verlag für Wissenschaft und Bildung, 2000 ; Heiko Weber et Maurizio di Bartolo dir., Jahrbuch für Europäische Wissenschaftskultur, n 3, 2007, Stuttgart, Franz Steiner Verlag, 2008.

28. Le Temps, 3, 8 et 12 août 1873.

29. Voir Theodor Nöldeke, Histoire littéraire de l'Ancien Testament, trad. fr. Hartwig Derenbourg et Jules Soury, Paris, Sandoz et Fischbacher, 1873; Hugo Magnus, Histoire de l'évolution du sens des couleurs, trad. fr. Jules Soury, Paris, C. Reinwald, 1878 ; Édouard Oscar Schmidt, Les Sciences naturelles et la philosophie de l'inconscient, trad. fr. Jules Soury et Édouard Meyer, Paris, GermerBaillière, 1879. Plus tard, il traduira et préfacera aussi l'ouvrage du physiologiste d'origine anglaise William Thierry Preyer, titulaire de la chaire de physiologie de Iéna; Wilhelm Preyer, Éléments de physiologie générale, tr. Jules Soury, Paris, Germer-Baillière-Félix Alcan, 1884.

30. Créée en 1872. Parmi ses fondateurs figurent Claude Bernard, Paul Broca et Armand de Quatrefages.

31. Il s'agit sans doute du Brébant, café-restaurant parisien situé 32 , boulevard Poissonnière, Paris $9^{e}$, fondé en 1865 et célèbre pour les dîners qu'y organisaient des personnalités et des membres de l'élite intellectuelle et artistique parisienne, dont Renan.

32. Voir la lettre de Soury à Haeckel du 22 août 1878. Parmi les amis cités on reconnaîtra l'ophtalmologue Édouard Meyer (1838-1902), l'anthropologue Gustave Le Bon (1841-1931), 
l'anthropologue Eugène Dally (1833-1887), la traductrice de Darwin Clémence Royer (1830-1902), le médecin Ernest Onimus (1840-1914) et l'éditeur Charles Reinwald (1812-1891).

33. Voir aussi : «Variétés. Un banquet transformiste", Revue Scientifique, $2^{\mathrm{e}}$ série, $1878, \mathrm{n}^{\circ} 15$, p. 211-212 ; «Discours de M. Haeckel », Le Temps, 30 août 1878. Voir aussi Linda Clark, op. cit., p. 91.

34. Ernst Haeckel, Les Preuves du transformisme. Réponse à Virchow par Ernest Haeckel, Paris, GermerBaillière, 1879. Avec une lettre du 18 décembre 1878, Soury avait envoyé les bonnes feuilles des Preuves du transformisme et annonçait qu'il avait déjà commencé le travail sur le Règne des Protistes. 35. Les discours de Haeckel et de Virchow avaient été publiés en français dans la Revue Scientifique du 8 décembre 1877 : Ernst Haeckel, «La théorie de l'évolution dans ses rapports avec la Philosophie naturelle ", La Revue scientifique de la France et de l'étranger. Revue des cours scientifiques ( $2^{\mathrm{e}}$ série), 8 décembre $1877, \mathrm{n}^{\circ} 13$, p. 529-534, suivi de la traduction du discours de Rudolf Virchow, «La liberté de la science dans l'État moderne », p. 534-543.

36. Virchow s'inquiète des possibles dérives scientistes de Haeckel: «Toute tentative pour transformer nos problèmes en propositions dogmatiques, pour présenter nos hypothèses comme le fondement de l'enseignement, la tentative notamment de déposséder l'Église et de remplacer simplement son dogme par une religion de la descendance, est condamnée à échouer, et dans son naufrage, elle ferait courir les plus grands périls à la situation qu'occupe la science. » (Ibid.)

37. Cette théorie très originale de Haeckel est l'une des toutes premières théories de l'hérédité. Elle sera traitée plus en détail dans la section dédiée à La Périgenèse des plastidules, le troisième essai traduit par Soury.

38. Virchow a toujours été très engagé en politique. Révolutionnaire en 1848 , il a été conseiller municipal à Berlin de 1859 à sa mort ; en 1861 il a été fondateur du parti progressiste allemand, Deutsche Fortschrittspartei ; en 1864 il est élu au Parlement de Prusse; en 1880 il sera élu au Parlement allemand.

39. Les Preuves du transformisme, op. cit., p. 97.

40. L'argument est un peu spécieux car cet enseignement vivant, fondé sur les hypothèses, est probablement plus adapté aux étudiants universitaires, ou du moins avancés, qu'aux élèves de l'école primaire ou secondaire, comme le fait remarquer le zoologue de Strasbourg Oscar Schmidt dans un appendice au volume.

41. Les Preuves du transformisme, op. cit., p. XV.

42. Ibid., p. XXXI.

43. Ibid., p.XXXV.

44. Ibid., p. XXXVI.

45. Ernst Haeckel, Le Règne des protistes, aperçu sur la morphologie des êtres vivants les plus inférieurs, suivi de la classification des protistes, Paris, Reinwald, 1879.

46. Amibes, Cellules ovulaires, Difflugies, Grégarines, Flagellés, Volvox, Noctiluques, Ciliés, Acinètes, Rhizopodes, Thalamophores, Radiolaires, Héliozoaires, Myxomycètes, Champignons, Diatomées, Labyrinthulées, Catallactes, Monères, Vibrions, Bactéries, et enfin «bathybius haeckeli » et monères.

47. «Introduction », p. VI-VII.

48. Premier chapitre : « Bory de Saint-Vincent et les Protistes », voir surtout p. XIII-XV.

49. Ibid., p. XIX, citant Claude Bernard, La Science expérimentale, Paris, Baillière, 1878, p. 178-182. Ce recueil contient aussi l'émouvant discours de Paul Bert lors des obsèques de Claude Bernard.

50. Ibid., p. XXVIII citant Cl. Bernard, op. cit., p. 188.

51. Ibid., p. XXXIII.

52. Ibid., p. XXXIII.

53. Ibid., p. XXXVI.

54. Ibid., p. XXXVII. 
55. La Science expérimentale, op. cit., p. 234 ; Leçons sur les phénomènes de la vie communs aux animaux et aux végétaux, Paris, Baillière, 1878, t. I, p. 192-195, 297, 320 et 349, cité dans «Introduction ", p. XLV.

56. «Introduction », p. XLVI citant Cl. Bernard, op. cit., p. 67.

57. Ernst Haeckel, Essais de psychologie cellulaire, Paris, Germer-Baillière, 1880.

58. Ibid., p. XX-XXI.

59. La transcription de cette lettre est donnée en annexe 1 . Soury ne se prive pas d'y critiquer l'éditeur Reinwald pour son avarice et pour les nombreuses fautes d'impression qu'il a constatées dans le Règne des Protistes.

60. Paul Bert (1833-1886), médecin et physiologiste, a été député radical gambettiste, et en 1881-1882 ministre de l'Instruction et des Cultes. Il est, avec Jules Ferry, le fondateur de l'« école gratuite, laïque et obligatoire » républicaine. Il a aussi été un vulgarisateur de la biologie et de l'anthropologie dans son journal La République et dans plusieurs manuels scolaires. Il fit inscrire sur sa stèle funéraire : «Science » et « Patrie ». Après sa mort, sa veuve demanda à Soury d'écrire sa biographie ; Soury refusa, s'estimant incapable d'écrire la biographie d'un optimiste.

61. Extrait d'une lettre de Renan du 19 décembre 1879 à Max Müller, Euvres complètes de Renan, Paris, Calmann-Lévy, 1961, t. X (correspondance), p. 817.

62. Dans cette dernière, Soury traite, assez brièvement, des doctrines de Haeckel concernant l'âme cellulaire et conclut, non sans humour: Hoc unum satis erit dictum, ex quo intelligi possit cellulares animas irridere facilius esse quam difficilem vite sensusque quastionem solvere. (De hylozoismo apud recentiores, G. Charpentier, 1881, p. 117 : Il suffira de dire ceci pour faire comprendre qu'il est plus facile de se moquer des âmes cellulaires que de résoudre le difficile problème de la vie et du psychisme.) Dans une lettre du 8 juillet 1881, Soury demande à Haeckel s'il a reçu ce volume.

63. Bréviaire de l'histoire du matérialisme, Paris, G. Charpentier, 1881. Ce bréviaire, inspiré de l' Histoire du matérialisme de Lange, sera suivi d'un recueil d'essais, Philosophie naturelle, Paris, G. Charpentier, 1882, témoignage de son adhésion au monisme évolutionniste haeckelien.

64. Théodule Ribot (1839-1916) convoitait aussi cette place, pour laquelle il se croyait autrement plus qualifié que «ce farceur de Soury », et il ne pardonna jamais à Paul Bert de ne pas l'avoir nommé. Voir Serge Nicolas, «L'introduction de l'enseignement de la psychologie scientifique en France ; Théodule Ribot (1839-1916) à la Sorbonne (1885) ", L'Année psychologique, 2000, n 100(2), p. 285-331.

65. Anatole France se réfère ici à l'ouvrage Les Fonctions du cerveau, Paris, F. Alcan, 1892. Texte extrait de Anatole France, «La vie littéraire. M. Jules Soury », Le Temps, 8 novembre 1891, cité par Jules Soury, Campagne nationaliste, op. cit., p. 47.

66. La transcription et la reproduction de cette lettre sont données en annexe 2.

67. En 1899 il publiera le manifeste moniste Die Welträthsel (Les énigmes de l'Univers), et en 1906 il sera le principal fondateur du Deutscher Monistenbund (Ligue moniste allemande).

68. Le livre sera traduit en français par Georges Vacher de Lapouge (1897), voir note 2.

69. La transcription et la reproduction de cette lettre sont données en annexe 3.

70. Le Système nerveux central, op. cit., 1899. Cette somme avait été précédée par son ouvrage déjà cité sur Les Fonctions du cerveau (1892), et pour une très longue entrée "Cerveau » dans le Dictionnaire de physiologie de Charles Richet, Paris, Alcan, 1896.

71. Campagne nationaliste, op. cit., p. 63.

72. Le Système nerveux central, op. cit., p. 1760-1782.

73. Ibid., p. 1778.

74. Ibid., p. 1779.

75. Sur l'antisémitisme de Soury, voir Toby Gelfand, „From religious to bio-medical antisemitism : the career of Jules Soury", Ann La Berge et Mordechai Feingold dir., French medical culture in the nineteenth century, Amsterdam et Atlanta, Rodopi, 1994, p. 248-279 ; Zeev Sternhell, "Jules Soury, un raciste français", Les Collections de l'Histoire, 1998, n³, p. 12 ; Pierre-André 
Taguieff, La Couleur et le Sang. Doctrines racistes à la française, Paris, Mille et une Nuits, Fayard, deuxième édition augmentée, 2002 op. cit., p. 150-172, et «L'invention racialiste du Juif », Raisons politiques, 2002, n 5, p. 29-51. Daniel Gasman dédie aussi un chapitre à Soury dans son livre controversé, Haeckel's monism and the birth of fascist ideology, New York, Peter Lang, 1998, p. 102-133. Toutefois le manque de contextualisation et la tendance de l'auteur à voir en Haeckel le seul responsable du fascisme, du nazisme et de l'Holocauste rendent cet ouvrage confus et peu fiable, en dépit de l'ampleur des recherches et de la richesse des sources.

76. Analysé par Marcel Gauchet, L'Inconscient cérébral, op. cit.

77. Campagne nationaliste, op. cit.

78. Ibid., p. 65.

79. Maurice Barrès, Mes Cahiers, vol. I, Janvier 1886-Novembre 1904, préface d'Antoine Compagnon, Paris, Éditions des Équateurs, 2010, p. 72.

80. À ce propos, Mousson-Lanauze (op.cit., 1927) cite l'épisode suivant: Soury avait confié son testament à Clemenceau; quand le «Tigre » connut l'attitude de Soury, il le lui renvoya avec les mots suivants: «Puisque vous avez changé d'idées, vous devez souhaiter un autre détenteur de vos volontés suprêmes ». Soury répondit «Je n'ai pas changé d'idées du tout »; et il lui retourna le testament.

81. Mes Cahiers, op. cit., vol. 2, p. 289.

82. La transcription et la reproduction de cette lettre sont données en annexe 4.

83. Parmi ses amis, seuls Barrès (L'Écho de Paris, $n^{\circ}$ du 14 août) et Charles Maurras lui rendent hommage. Charles Maurras, "Jules Soury", Action française, 13 août 1915 ; publié aussi dans Tombeaux, Paris, Nouvelle Librairie Nationale, 1921, p. 80-85. Soury a néanmoins droit aux nécrologies institutionnelles d'usage et même à une notice dans le British Medical Journal. Voir Marius Sepet, "Jules Soury", Bibliothèque de l'École des Chartes, 1915, nº 76, p. 455-456; Paul Souday, nécrologie de J. Soury dans Le Temps du 16 août 1915 ; nécrologie "Jules Soury ", École pratique des hautes études, Section des sciences historiques et philologiques. Annuaire 1915-1916, Paris, Imprimerie nationale, 1915, p. 71-73 ; nécrologie signée Junius, dans L'Écho de Paris, 16 août 1915 ; «M. Jules Soury », British Medical Journal, 1915, n² 2, p. 491.

84. Le Stupide XIX $X^{e}$ siècle. Exposé des insanités meurtrières qui se sont abattues sur la France depuis 130 ans, 1789-1919, Paris, Nouvelle Librairie Nationale, 1922.

85. Citation du célèbre passage de Virgile (Énéide, I, 118) qui décrit le naufrage d'Énée : « de rares nageurs dans le vaste abîme ».

\section{RÉSUMÉS}

Chartiste parisien, Jules-Auguste Soury (1842-1915) a traduit en français trois ouvrages de l'éminent zoologue darwiniste allemand Ernst Haeckel (1834-1919) : Les Preuves du transformisme (Germer Baillière, 1879), Le Règne des protistes (Reinwald, 1879) et Essais de psychologie cellulaire (Germer Baillière, 1880). Les archives du Ernst-Haeckel-Haus à Iéna conservent trente-cinq lettres inédites adressées par Soury à Haeckel entre 1878 et 1914. Ce corpus permet de suivre quelques étapes clés de la vie de Soury, comme l'échec de « sa » chaire d'Histoire des Religions au Collège de France et sa nomination par son mentor Paul Bert à l'École des Hautes Études, où il enseignera l'histoire de la psychologie physiologique et rédigera son opus magnum, Le Système nerveux central (1899) ; il confirme par ailleurs l'interprétation rigidement mécaniste, matérialiste et athée des 
théories haeckeliennes par Soury, déjà évidente dans les longues préfaces qu'il rédige pour ses traductions. Ces lettres illustrent également l'évolution des relations entre le traducteur et l'auteur, allant de l'admiration à la déception. Lorsque Haeckel lui envoie son livre Der Monismus, Soury rédigera une virulente lettre de rupture (27 décembre 1892), dans laquelle il affirme ne plus pouvoir suivre le maître dans sa nouvelle religion moniste, "avec sa trinité du Beau, du Vrai, du Bien ». Dans sa dernière lettre, envoyée en 1914 à l'occasion du 80e anniversaire de Haeckel, après un long intervalle et juste avant l'éclatement de la Grande Guerre, Soury évoquera toutefois le souvenir nostalgique du temps passé à ses traductions, de celles qui furent «ses meilleures heures, des heures de travail et de méditation ».

Jules-Auguste Soury (1842-1915), a Parisian scholar trained at the École des Chartes, authored the French translations of three books by the famous German zoologist and darwinist Ernst Haeckel (1834-1939) : Les Preuves du transformisme (Germer Baillière, 1879), Le Règne des protistes (Reinwald, 1879) and Essais de psychologie cellulaire (Germer Baillière, 1880). The archives of the Ernst-HaeckelHaus in Jena retain 35 unpublished letters sent by Soury to Haeckel between 1878 and 1914, illustrating some milestones in Soury's life such as his failed attempt at obtaining "his" chair of History of religions at the Collège de France, or his subsequent nomination by his mentor Paul Bert at the École des Hautes Études, where he taught the history of "physiological psychology" and wrote his major work, Le Système nerveux central (1899). The letters confirm the strictly mechanistic, materialistic ant atheistic interpretation of Haeckel's theories by Soury, that is already evident from his lengthy prefaces to the translations. They also show the evolution of the relationship between the translator and the author, from initial admiration to disillusion: upon reception of Haeckel's book on Monism, Soury wrote a virulent break-up letter (December 27, 1892) asserting that he could not follow his master in his new monist religion, "with its trinity of the Beautiful, the True and the Good". In his last letter sent to Haeckel in 1914 for his 80th birthday, after a long time interval and immediately before the Great War, Soury will nevertheless bring back his nostalgic memories of the time spent in translating, of those that had been "his best hours, hours of work and of meditation".

\section{INDEX}

Keywords : Haeckel (Ernest), Soury (Jules-Auguste), correspondence, Darwinism, transformism, recapitulation, monism, materialism, free thought, cell theory, protists, cell psychology,

heredity, soul, hylozoism, anti-semitism

Mots-clés : Haeckel (Ernest), Soury (Jules-Auguste), correspondance, darwinisme, transformisme, récapitulation, monisme, matérialisme, libre pensée, théorie cellulaire, protistes, psychologie cellulaire, hérédité, âme, hylozoïsme, antisémitisme

\section{AUTEUR}

\section{LAURA BOSSI}

CNRS - UMR 7219 Laboratoire SPHERE 